\title{
Revisiting Asynchronous Linear Solvers: Provable Convergence Rate Through Randomization*
}

\author{
Haim Avron \\ IBM T.J. Watson Research Center \\ haimav@us.ibm.com
}

\author{
Alex Druinsky \\ Lawrence Berkeley National Laboratory \\ adruinsky@lbl.gov
}

\author{
Anshul Gupta \\ IBM T.J. Watson Research Center \\ anshul@us.ibm.com
}

\begin{abstract}
Asynchronous methods for solving systems of linear equations have been researched since Chazan and Miranker's pioneering 1969 paper on chaotic relaxation. The underlying idea of asynchronous methods is to avoid processor idle time by allowing the processors to continue to make progress even if not all progress made by other processors has been communicated to them.

Historically, the applicability of asynchronous methods for solving linear equations was limited to certain restricted classes of matrices, such as diagonally dominant matrices. Furthermore, analysis of these methods focused on proving convergence in the limit. Comparison of the asynchronous convergence rate with its synchronous counterpart and its scaling with the number of processors were seldom studied, and are still not well understood.

In this paper, we propose a randomized shared-memory asynchronous method for general symmetric positive definite matrices. We rigorously analyze the convergence rate and prove that it is linear, and is close to that of the method's synchronous counterpart if the processor count is not excessive relative to the size and sparsity of the matrix. We also present an algorithm for unsymmetric systems and overdetermined least-squares. Our work presents a significant improvement in the applicability of asynchronous linear solvers as well as in their convergence analysis, and suggests randomization as a key paradigm to serve as a foundation for asynchronous methods.
\end{abstract}

\section{Introduction}

It has long been recognized that high global synchronization costs will eventually limit the scalability of iterative solvers. So early on, starting with the pioneering work of Chazan and Miranker on chaotic relaxation in 1969 [6] (see review by Frommer and Szyld [8]), asynchronous methods have been researched and deployed. These methods avoid synchronization points and their associated costs by allowing processors to continue to work even if not all progress made by other processors has been communicated to them.

While asynchronous methods were successfully applied to many numerical problems [8], interest in them dwindled over the years. One important reason is that until recently, concurrency was not large enough to warrant the use of asynchronous methods, as asynchronous methods typically require more computation when compared to their synchronous counterparts. Other reasons are related to the limits of existing theory on asynchronous methods. Historically, the applicability of asynchronous methods for solving linear equations was limited to restricted classes of matrices, such as diagonally dominant matrices. This had a substantially negative impact on the relevance and interest in asynchronous

\footnotetext{
${ }^{*}$ An extended abstract of this work appears in the proceedings of the 28th IEEE International Parallel \& Distributed Processing Symposium (IPDPS), 2014 [1].
} 
methods, as most of the matrices arising in applications did not posses the required attributes. Furthermore, analysis of asynchronous methods for solving linear equations focused on proving convergence in the limit. How the rate of convergence compares to the rate of convergence of the synchronous counterparts, and how this rate scales when the number of processors increases, was seldom studied and is still not well understood. It was observed experimentally that asynchronous methods can sometimes be substantially slower than their synchronous counterparts [5].

Today, as we push towards extreme scale systems, asynchronous algorithms are becoming more and more attractive. In addition to the high synchronization costs due to massive parallelism, other hardware issues make asynchronous methods attractive as well. Current hardware trends suggest that software running on extreme-scale parallel platforms will be expected to encounter and be resilient to nondeterministic behavior from the underlying hardware. Asynchronous methods are inherently well-suited to meet this challenge. On the other hand, it is also clear that a paradigm shift regarding the way asynchronous methods are designed and analyzed must be made, if such methods are to be deployed. To that end, this paper makes three significant contributions. It presents an asynchronous solver with randomization as a key algorithmic component, a rigorous analysis that affirms the role of randomization as an effective tool for improving asynchronous solvers, and an analytical methodology for asynchronous linear solvers based on a realistic bounded-delay model.

Specifically, we present a new asynchronous shared-memory parallel solver for symmetric positive definite matrices with a provable linear convergence rate under a mostly asynchronous computational model that assumes bounded delays. A key component of our algorithm is randomization, which allows the processors to make progress independently with only a small probability of interfering with each other. Our analysis shows a convergence rate that is linear in the condition number of the matrix, and depends on the number of processors and the degree to which the matrix is sparse. A slightly better bound is achieved if we occasionally synchronize the processors. In either case, as long as the number of processors is not too large relative to the size and sparsity of the matrix, the convergence rate is close to that of the synchronous counterpart. Unlike in general asynchronous methods, the convergence rate does not depend on numerical classification of the matrix (e.g., diagonal dominance). In particular, our method will converge for essentially any large sparse symmetric positive definite matrix as long as not too many processors are used. We also present an algorithm for unsymmetric systems and overdetermined least-squares.

Our method and its analysis do have some limitations. Adapting the algorithm to the distributed memory setting is not straightforward. Our algorithm allows each processor to update all the entries of the solution vector, but in a distributed memory setting it is desirable that each processor owns and be the sole updater of only a subset of the entries. To allow this, a more limited form of randomization should be used, and this is not explored in the paper. Our algorithm also tends to generate much more cache misses than classical asynchronous methods for structured matrices. Again, it may be possible to circumvent this using a more restricted form of randomization. More importantly, our algorithm's convergence is inherently slower than that of Krylov-subspace methods, which is a feature of the underlying synchronous algorithm. For this reason, the algorithm is most suitable when only moderate accuracy is sought, either when we require low accuracy in the ultimate solution or when we use the algorithm as a preconditioner in a flexible Krylov method. Our algorithm relies on some assumptions that are hard to enforce in practice, and the convergence results have a parameter which is hard to quantify better than by just a very rough upper bound. Finally, we remark that bounds tend to be rather pessimistic (this is true also for the synchronous algorithm that is the basis for our algorithm).

Nevertheless, even with these limitations we believe our work presents a significant improvement in the applicability of asynchronous linear solvers, as well as in the convergence analysis, and suggests randomization as a key paradigm to serve as a foundation for asynchronous methods.

While the primary aim of this paper is to present analytical results, we also include some experimental results. With our implementation, we are able to demonstrate that the proposed method can be attractive for certain types of linear systems even in the absence of massive parallelism. Previous asynchronous methods, as well as ours, are based on basic iteration (e.g., Gauss-Seidel). Those are known to convergence very slowly in the long run when compared with Krylov subspace methods. However, big data applications typically require very low accuracy, so they are better served using basic iterations as these tend to initially converge very quickly and scale better. Our experiments show that for a linear system arising from analysis of social media data, our proposed algorithm scales well, pays very little to no penalty for asynchronicity, and overall seems to present the best choice for solving the said linear system to the 
required accuracy.

We review related work in Section 2. Essential background on Randomized Gauss-Seidel is given in Section 3 . In Section 4 we propose two asynchronous models for executing Randomized Gauss-Seidel: one assumes that consistent reads have been enforced, another does not. Section 5 analyzes the convergence when the consistent read assumption is enforced. Section 6 shows that convergence can be improved if we control the step-size. In Section 7, we analyze convergence rate when we allow inconsistent reads. We briefly discuss unsymmetric systems and overdetermined least-squares in Section 8. Section 9 presents experimental results. Finally, in Section 10 we make some concluding remarks and discuss future work.

\section{Setup and Notation}

Most of this paper is concerned with solving the linear equation $\mathbf{A x}=\mathbf{b}$ where $\mathbf{A} \in \mathbb{R}^{n \times n}$ is a symmetric positive definite matrix, and $\mathbf{b} \in \mathbb{R}^{n}$. For simplicity we assume that $\mathbf{A}$ has a unit diagonal. This is easily accomplished using re-scaling. Our results can be easily generalized to allow an arbitrary diagonal, but making this assumption helps keep the presentation and notation more manageable. We denote the exact solution to this equation by $\mathbf{x}^{\star}$, i.e. $\mathbf{x}^{\star}=\mathbf{A}^{-1} \mathbf{b}$. We denote the largest eigenvalue of $\mathbf{A}$ by $\lambda_{\max }$, and the smallest eigenvalue by $\lambda_{\min }$. The condition number of $\mathbf{A}$, which is equal to $\lambda_{\max } / \lambda_{\min }$, is denoted by $\kappa$.

We are predominantly interested in the case where $\mathbf{A}$ is sparse and very large, and the number of non-zeros in each row is between $C_{1}$ and $C_{2} \ll n$ with a small ratio between $C_{2}$ and $C_{1}$. This scenario frequently occurs in many scientific computing applications. Throughout the paper we refer to this scenario as the reference scenario. We state and prove more general results; we do not use the properties of the reference scenario in the proofs. The reference scenario is mainly useful for the interpretation of the practical implications of the results. Note that in the reference scenario we have $\lambda_{\max } \leq C_{2} \ll n$, as $\mathbf{A}$ has a unit-diagonal (so off-diagonal entries must be smaller than or equal to one).

We use $(\cdot, \cdot)_{\mathbf{A}}$ to denote the $\mathbf{A}$ inner product. That is, $(\mathbf{x}, \mathbf{y})_{\mathbf{A}} \equiv \mathbf{y}^{\mathrm{T}} \mathbf{A} \mathbf{x}$ where $\mathbf{x}, \mathbf{y} \in \mathbb{R}^{n}$. The fact that $\mathbf{A}$ is a symmetric positive definite matrix guarantees that $(\cdot, \cdot)_{\mathbf{A}}$ is an inner product. The $\mathbf{A}$-norm is defined by $\|\mathbf{x}\|_{\mathbf{A}} \equiv$ $\sqrt{(\mathbf{x}, \mathbf{x})_{\mathbf{A}}}$. We use $\mathbf{e}^{(1)}, \mathbf{e}^{(2)}, \ldots, \mathbf{e}^{(n)}$ to denote the $n$-dimensional identity vectors (i.e. $\mathbf{e}^{(i)}$ is one at position $i$ and zero elsewhere). $\mathbf{A}_{i}$ denotes row $i$ of $\mathbf{A}$, and $\mathbf{A}_{i j}$ denotes the $i, j$ entry of $\mathbf{A}$. We will generally use subscript indices on vectors for iteration counters. The notation $(\mathbf{x})_{i}$ denotes the $i$ th entry of $\mathbf{x}$.

Throughout the paper we describe algorithms that generate a series of approximations to $\mathbf{x}^{\star}$, denoted by $\mathbf{x}_{0}, \mathbf{x}_{1}, \ldots$ (subscript index is the iteration counter), which are actually random vectors. We denote the expected squared $\mathbf{A}$-norm of the error of $\mathbf{x}_{m}$ by $E_{m}$. That is,

$$
E_{m} \equiv \mathbb{E}\left[\left\|\mathbf{x}_{m}-\mathbf{x}^{\star}\right\|_{\mathbf{A}}^{2}\right] .
$$

\section{Related Work}

Asynchronous methods were first suggested by Chazan and Miranker [6] in their pioneering paper on chaotic relaxation. The theory and application of asynchronous iterations has since been studied and used by many authors. Noteworthy is the seminal text by Bertsekas and Tsitsiklis [4]. A more recent review is by Frommer and Szyld [8].

Historically, work on asynchronous methods focused on proving that the methods converge in the limit, and not on convergence rate analysis. In particular, the relation to the convergence rate of synchronous counterparts, and the scaling of these methods, were seldom studied. We are aware of only two exceptions of work published before 2011, but the results are unsatisfactory. Baudet [3] generalizes the Chazan-Miranker result to nonlinear mappings using the same model of asynchronism. For the linear case, he does not extend the class of systems that can be solved in this model (the Chazan-Miranker result is an if and only if result, so there is no possibility for improvement in that model in that respect), but he does show that given a trace of an asynchronous iteration, if in that trace the maximum delay on purging old information and the rate of updating all components is bounded, then convergence is linear with the rate of convergence divided by the sum of these two bounds. The author does not analyze and show rigorous convergence rates for a concrete algorithm for solving a linear system, nor compare the convergence rate of some 
specific asynchronous algorithm to its synchronous counterpart. Bertsekas and Tsitsiklis [4, Section 7.2, Exercise 1.2] prove a linear convergence rate of certain asynchronous iterations for some classes of matrices (like weakly diagonally dominant matrices), but analyze how the rate of convergence depends on the measure of asynchronism only under very restrictive conditions and in a hard to interpret manner [4, Section 6.3.5].

Following the influential work of Niu et al. [15], recent work has focused on analyzing the rate of convergence. When discussing these recent results it is important to distinguish between methods that assume a consistent read model, and models that allow inconsistent reads. Informally (we give a formal definition in Section 4), the consistent read model assumes that the part of the state (i.e., iteration vector) that the algorithm reads in order to update the state is consistent with a state that existed in shared memory at some point in time. Without special provisions, which might be computationally expensive, the consistent read assumption is somewhat unsatisfactory, although the analysis of a consistent read model is not without merit, as we explain in Section 4

The basic model proposed by Niu et al. [15] is as follows. There is an iteration vector $\mathbf{x}$ that is stored in sharedmemory. All processors share this memory and update it in an asynchronous, uncoordinated fashion, without any form of locking. This implies that the version of $\mathbf{x}$ that is used by a processor to update $\mathbf{x}$ is not the same as the version on which this update is applied, as $\mathbf{x}$ has possibly been updated by other processors in the interim. It is however assumed that write operations are atomic, and that there is a bound $\tau$ on how many updates are missed. This model matches modern multicore architectures well. Under an additional assumption of consistent reads, Niu et al. establish a sublinear convergence rate of asynchronous stochastic gradient descent. Our model follows the one proposed by Niu et al. [15], although we analyze convergence both in the consistent read and inconsistent read model. We also establish a linear convergence rate, unlike the sublinear rate for stochastic gradient descent, with a better dependence on $\tau$.

Liu et al. [14] propose an Asynchronous Randomized Kaczmarz algorithm for solving consistent square and overdetermined linear systems. They use the same model as Niu et al. do, and assume consistent reads as well. A linear convergence rate is established. An extension to inconsistent systems is discussed as well.

Later, Liu et al. [13] develop an asynchronous stochastic coordinate descent algorithm. Again, they use the same model as Niu et al., and continue to assume consistent reads. Furthermore, they assume that the radius of the iterate set is bounded, which is a condition that might be hard to enforce in an asynchronous linear solver. They prove a sublinear $(1 / m)$ convergence on general convex functions and a linear convergence rate on functions that satisfy an "essential strong convexity" property.

More recently, Liu et al. [12] suggest an asynchronous stochastic proximal coordinate-descent algorithm for composite objective functions. They allow inconsistent reads, and prove linear convergence for optimally strongly convex functions, and a sublinear rate for general smooth convex functions.

Our algorithm is closely related to the stochastic coordinate descent algorithm in the sense that in essence our algorithm is an asynchronous stochastic coordinate descent method applied to the strongly convex quadratic optimization problem $\min _{\mathbf{x}} \frac{1}{2} \mathbf{x}^{\mathrm{T}} \mathbf{A} \mathbf{x}-\mathbf{b}^{\mathrm{T}} \mathbf{x}$. However, our results are much more tuned and interpretable to the problem we consider $(\mathbf{A x}=\mathbf{b})$ than the convergence result for general strongly convex functions.

Hook and Dingle [10] analyze the convergence of the Jacobi iteration when it is executed asynchronously on a distributed memory machine. They prove upper and lower bounds on the convergence rate of the iteration that are formulated in terms of the spectral radius of $\mathbf{A}$ and two parameters of the asynchronous execution dynamics. Their results indicate when convergence takes place and how fast it is, even without the help of randomization. The dependence of the bounds on parameters of the execution dynamics makes the convergence guarantee hard to interpret. Nevertheless, the results show that performance can suffer if an entry of the iterate is repeatedly updated using stale data because of a slow communication link or fails to be updated at all because of a slow processor. This indicates the potential of using randomization to obtain robust performance in the face of such single-point-of-failure vulnerabilities.

Unrelated to the previous, we also note Freris and Zouzias's [7] work on using an asynchronous variant of randomized Kaczmarz [20] to synchronize clocks in a wireless network. They analyze the convergence rate in a semiasynchronous model that is suitable for wireless networks, but not for shared-memory numerical computations. 


\section{Randomized Gauss-Seidel}

Our asynchronous algorithm is based on the randomized variant of the Gauss-Seidel iteration, originally proposed by Leventhal and Lewis [11]. We actually use a slight modification due to Griebel and Oswald [9] that introduces a stepsize (akin to under- and over-relaxation). The goal of this section is to describe and review the basic properties of the randomized Gauss-Seidel iteration.

Consider the following iteration applied to some arbitrary initial vector $\mathbf{x}_{0} \in \mathbb{R}^{n}$, and a series of direction vectors $\mathbf{d}_{0}, \mathbf{d}_{1}, \ldots$ :

$$
\begin{aligned}
\mathbf{r}_{j} & =\mathbf{b}-\mathbf{A} \mathbf{x}_{j} \\
\gamma_{j} & =\mathbf{d}_{j}^{\mathrm{T}} \mathbf{r}_{j} \\
\mathbf{x}_{j+1} & =\mathbf{x}_{j}+\beta \gamma_{j} \mathbf{d}_{j},
\end{aligned}
$$

where $0<\beta<2$. In terms of the analysis it is more convenient to write the iteration in the following equivalent form:

$$
\begin{aligned}
\gamma_{j} & =\left(\mathbf{x}^{\star}-\mathbf{x}_{j}, \mathbf{d}_{j}\right)_{\mathbf{A}} \\
\mathbf{x}_{j+1} & =\mathbf{x}_{j}+\beta \gamma_{j} \mathbf{d}_{j} .
\end{aligned}
$$

Both iterations are listed to show that even though the unknown $\mathrm{x}^{\star}$ appears in (1), the iteration is computable.

In (1) the scalars $\gamma_{0}, \gamma_{1}, \ldots$ are selected so as to minimize $\left\|\mathbf{x}^{\star}-\mathbf{x}_{j+1}\right\|_{\mathbf{A}}$ when $\mathbf{x}_{j+1}$ is obtained from $\mathbf{x}_{j}$ by taking a step in the direction $\mathbf{d}_{j}$ with $\beta=1$. There are quite a few ways to set $\mathbf{d}_{0}, \mathbf{d}_{1}, \ldots$ Each is associated with a different per-iteration cost, and different convergence properties. One well known method is setting $\mathbf{d}_{i}=\mathbf{e}^{((i \bmod n)+1)}$. In that case, every $n$ iterations corresponds to a single iteration of Gauss-Seidel (recall that we assume that the matrix has unit diagonal).

Leventhal and Lewis suggested using random directions instead of deterministic ones: $\mathbf{d}_{0}, \mathbf{d}_{1}, \ldots$ are i.i.d. random vectors, taking $\mathbf{e}^{(1)}, \ldots, \mathbf{e}^{(n)}$ with equal probability 1 . For this distribution of direction vectors, Griebel and Oswald [9] prove the following bound on the expected error in the A-norm (the case of $\beta=1$ was analyzed by Leventhal and Lewis [11]):

$$
E_{m} \leq\left(1-\frac{\beta(2-\beta) \lambda_{\min }}{n}\right)^{m}\left\|\mathbf{x}_{0}-\mathbf{x}^{\star}\right\|_{\mathbf{A}}^{2} .
$$

So, the randomized Gauss-Seidel iteration converges in expectation at a linear rate $\underbrace{2}$. Markov's inequality now implies that given $\epsilon>0$ and $\delta \in(0,1)$, for

$$
m \geq \frac{n}{\beta(2-\beta) \lambda_{\min }} \ln \left(\frac{1}{\delta \epsilon^{2}}\right)
$$

we have

$$
\operatorname{Pr}\left(\left\|\mathbf{x}_{m}-\mathbf{x}^{\star}\right\|_{\mathbf{A}} \geq \epsilon\left\|\mathbf{x}_{0}-\mathbf{x}^{\star}\right\|_{\mathbf{A}}\right) \leq \delta .
$$

If we could compute $\left\|\mathbf{x}_{m}-\mathbf{x}^{\star}\right\|_{\mathbf{A}}$, this will imply a randomized algorithm whose probabilistic guarantees are only on the running time, and not on the quality of approximation. In practice, we can check the residual $\left\|\mathbf{b}-\mathbf{A} \mathbf{x}_{m}\right\|_{2}$, as is typically done in iterative methods. Similar transformations can be done to other bounds throughout this paper. These transformations are rather technical, so we omit them. Note that the expected cost per iteration of randomized GaussSeidel is $\Theta(\mathbf{n n z}(\mathbf{A}) / n)$, so $n$ iterations (which we refer to as a sweep) are about as costly as a single Gauss-Seidel iteration.

The proof of (2) relies on the following lemma, which we use extensively in our analysis as well. The upper bound in the lemma was not proven by Leventhal and Lewis [11], but it can be proved using the same technique they used to prove the lower bound. For completeness we include a proof.

\footnotetext{
${ }^{1}$ Leventhal and Lewis consider the more general setting where A does not have unit diagonal. For that case, they analyze non-uniform probabilities. When the matrix has unit diagonal, their algorithm and the convergence analysis reduces to the ones stated here.

${ }^{2}$ Some care should be employed with terminology. Some mathematicians or computer scientists might say this is an exponential or geometric convergence rate. However, numerical analysts refer to this rate as linear, as it is linear in $O(\log (\epsilon))$ where $\epsilon$ is the desired reduction factor of the error.
} 
Lemma 1. Let $\mathbf{d}$ be a random vector taking $\mathbf{e}^{(1)}, \ldots, \mathbf{e}^{(n)}$ with equal probability. Suppose that $\mathbf{x}$ and $\mathbf{d}$ are independent. Then,

$$
\frac{\lambda_{\min }}{n} \mathbb{E}\left[\left\|\mathbf{x}-\mathbf{x}^{\star}\right\|_{\mathbf{A}}^{2}\right] \leq \mathbb{E}\left[\left(\mathbf{x}-\mathbf{x}^{\star}, \mathbf{d}\right)_{\mathbf{A}}^{2}\right] \leq \frac{\lambda_{\max }}{n} \mathbb{E}\left[\left\|\mathbf{x}-\mathbf{x}^{\star}\right\|_{\mathbf{A}}^{2}\right] .
$$

Proof. Let $\mathbf{B}$ be the unique symmetric positive matrix such that $\mathbf{A}=\mathbf{B}^{2}$. We have

$$
\begin{aligned}
\mathbb{E}\left[\left(\mathbf{x}-\mathbf{x}^{\star}, \mathbf{d}\right)_{\mathbf{A}}^{2}\right] & =\mathbb{E}\left[\mathbb{E}\left[\left(\mathbf{x}-\mathbf{x}^{\star}, \mathbf{d}\right)_{\mathbf{A}}^{2}\right] \mid \mathbf{x}\right] \\
& =\mathbb{E}\left[\frac{1}{n} \sum_{i=1}^{n}\left(\mathbf{x}-\mathbf{x}^{\star}, \mathbf{e}_{i}\right)_{\mathbf{A}}^{2}\right] \\
& =\frac{1}{n} \mathbb{E}\left[\left\|\mathbf{A}\left(\mathbf{x}-\mathbf{x}^{\star}\right)\right\|_{2}^{2}\right] \\
& =\frac{1}{n} \mathbb{E}\left[\left(\mathbf{x}-\mathbf{x}^{\star}\right)^{\mathrm{T}} \mathbf{A}^{2}\left(\mathbf{x}-\mathbf{x}^{\star}\right)\right] \\
& =\frac{1}{n} \mathbb{E}\left[\frac{\left(\mathbf{x}-\mathbf{x}^{\star}\right)^{\mathrm{T}} \mathbf{B} \mathbf{A B}\left(\mathbf{x}-\mathbf{x}^{\star}\right)}{\left(\mathbf{x}-\mathbf{x}^{\star}\right)^{\mathrm{T}} \mathbf{B B}\left(\mathbf{x}-\mathbf{x}^{\star}\right)} \cdot\left(\mathbf{x}-\mathbf{x}^{\star}\right)^{\mathrm{T}} \mathbf{B B}\left(\mathbf{x}-\mathbf{x}^{\star}\right)\right] \\
& =\frac{1}{n} \mathbb{E}\left[\frac{\left(\mathbf{x}-\mathbf{x}^{\star}\right)^{\mathrm{T}} \mathbf{B} \mathbf{A B}\left(\mathbf{x}-\mathbf{x}^{\star}\right)}{\left(\mathbf{x}-\mathbf{x}^{\star}\right)^{\mathrm{T}} \mathbf{B B}\left(\mathbf{x}-\mathbf{x}^{\star}\right)} \cdot\left\|\mathbf{x}-\mathbf{x}^{\star}\right\|_{\mathbf{A}}^{2}\right] .
\end{aligned}
$$

According to the Courant-Fischer theorem, for every vector $\mathbf{y} \neq 0$ we have

$$
\lambda_{\min } \leq \frac{\mathbf{y}^{\mathrm{T}} \mathbf{A y}}{\mathbf{y}^{\mathrm{T}} \mathbf{y}} \leq \lambda_{\max }
$$

Applying the last inequality to the previous equality with $\mathbf{y}=\mathbf{B}\left(\mathbf{x}-\mathbf{x}^{\star}\right)$ completes the proof.

\section{Non-Unit Diagonal}

We now explain why there is no loss in generality in assuming that $\mathbf{A}$ has unit diagonal.

Suppose that B does not have unit diagonal. Consider the following more general Randomized Gauss-Seidel iteration (also due to Leventhal and Lewis [11]):

$$
\begin{aligned}
\tilde{\gamma}_{j} & =\frac{\left(\mathbf{y}^{\star}-\mathbf{y}_{j}, \mathbf{d}_{j}\right)_{\mathbf{B}}}{\left(\mathbf{d}_{j}, \mathbf{d}_{j}\right)_{\mathbf{B}}} \\
\mathbf{y}_{j+1} & =\mathbf{y}_{j}+\beta \tilde{\gamma}_{j} \mathbf{d}_{j} .
\end{aligned}
$$

where $\mathbf{y}^{\star}$ is the solution to $\mathbf{B y}=\mathbf{z}$, and $\mathbf{d}_{0}, \mathbf{d}_{1}, \ldots$ are i.i.d. random vectors taking $\mathbf{e}^{(1)}, \ldots, \mathbf{e}^{(n)}$ with equal probability. Let $\mathbf{D}$ be the diagonal matrix such $\mathbf{A}=\mathbf{D B D}$ has unit diagonal, and consider the unit-diagonal Randomized Gauss-Seidel iteration (1) for the linear system $\mathbf{A x}=\mathbf{D z}$ using the same direction vectors $\mathbf{d}_{0}, \mathbf{d}_{1}, \ldots$ It is not hard to verify that $\mathbf{y}_{j}=\mathbf{D} \mathbf{x}_{j}$ and that $\left\|\mathbf{x}_{j}-\mathbf{x}^{\star}\right\|_{\mathbf{A}}=\left\|\mathbf{y}_{j}-\mathbf{y}^{\star}\right\|_{\mathbf{B}}$. Therefore, analyzing the unit-diagonal scenario is sufficient.

\section{Asynchronous Randomized Gauss-Seidel (AsyRGS)}

Algorithm 1 contains a pseudo-code description of randomized Gauss-Seidel in which we made the read and update operations explicit. This obviously entails some details that are, in a sense, implementation specific. There are implementations of the randomized Gauss-Seidel iteration which do not match the description in Algorithm 1 .

Consider a shared memory model with $P$ processors. Each processor follows Algorithm 1 using the same $\mathbf{x}$, i.e. all processors read and update the same $\mathbf{x}$ stored in a shared memory. The processors do not explicitly coordinate or synchronize their iterations. We do, however, impose assumptions, some of which may require enforcement in an actual implementation. The first assumption is rather simple: the update operation in each iteration is atomic. 


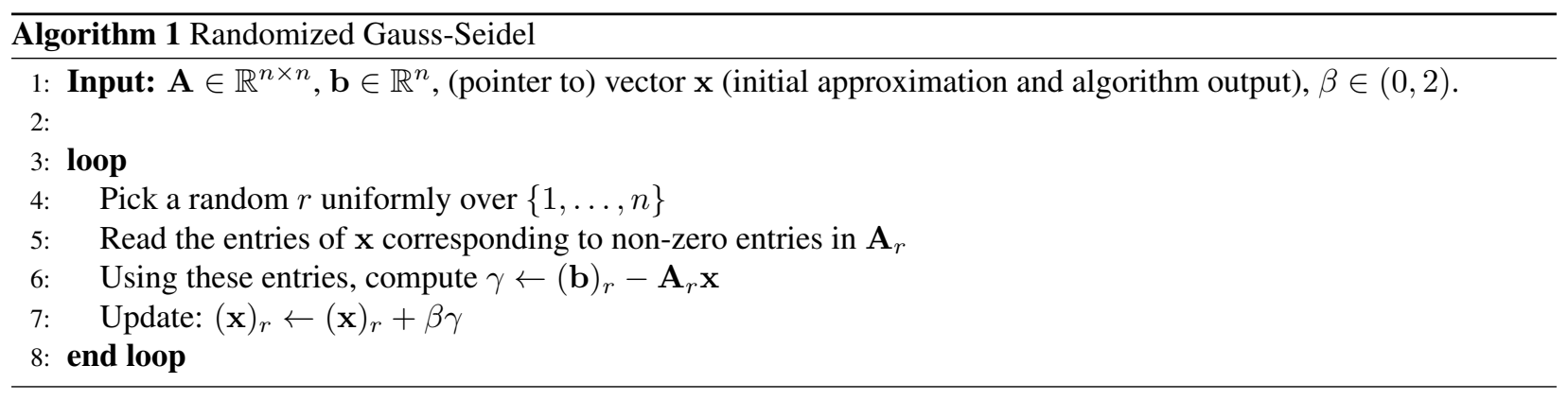

Assumption A-1 (Atomic Write). The update operation in line 7 is atomic.

The update operation operates on a single coordinate in $\mathrm{x}$. For single- or double-precision floating point numbers, updates of the form used in line 7 have hardware support on many modern processors (e.g. compare-and-exchange on recent Intel processors).

If atomic write is enforced, then for the sake of the analysis we can impose an order $\mathbf{x}_{0}, \mathbf{x}_{1}, \mathbf{x}_{2}, \ldots$ on the values that $\mathbf{x}$ takes during the computation. Here $\mathbf{x}_{j}$ denotes the value of $\mathbf{x}$ after $j$ updates have been applied (breaking ties in an arbitrary manner).

We now turn our attention to the read operation in line 5. Here we consider two possible models. In the first model, we assume the following consistent read assumption is enforced.

Assumption A-2 (Consistent Read). The values of the entries of $\mathrm{x}$ read in line 5 appeared together in $\mathrm{x}$ at some time before the update operation (line 7) is executed.

Note that Assumption A-2 does not necessarily imply that none of the entries read during the execution of line 5 are modified while that line is being executed; this is only one way of enforcing this assumption. More formally, if $R$ denotes the set of entries read in the execution of line 5 for a particular execution of the iteration, and $M$ denotes the set of entries modified during the execution of line 5 in that iteration, then Assumption A-2 holds for that iteration if $R \cap M=\emptyset$. However, this is only a sufficient condition, not a necessary one.

With consistent read, we can denote by $k(j) \leq j$ the maximum iteration index such $\mathbf{x}_{k(j)}$ is equal to the values read on line 5, on the indices read during the execution of line 5. The existence of such a $k(j)$ is guaranteed by Assumption A-2 (since all writes are atomic, all time intervals correspond to some iteration index). The iteration can then be written:

$$
\begin{aligned}
\gamma_{j} & =\left(\mathbf{x}^{\star}-\mathbf{x}_{k(j)}, \mathbf{d}_{j}\right)_{\mathbf{A}} \\
\mathbf{x}_{j+1} & =\mathbf{x}_{j}+\beta \gamma_{j} \mathbf{d}_{j} .
\end{aligned}
$$

We also consider a model where we allow inconsistent reads. Since every iteration changes a single coordinate, and we require all writes to be atomic, the value of $\mathbf{x}$ read in line 5 is the result of a subset of the updates that occurred before the write operation in line 7 is executed. Let us denote by $K(j) \subseteq\{0,1, \ldots, j-1\}$ a maximal set of updates consistent with the computation of $\gamma$ in iteration $j$. In other words, an index $i \leq j$ is in $K(j)$ if either it updates an entry of $\mathbf{x}$ not read for computing $\gamma_{j}$, or it updates an entry and the update was applied before that entry was read. The entries read are consistent with the vector

$$
\mathbf{x}_{K(j)}=\mathbf{x}_{0}+\sum_{i \in K(j)} \beta \gamma_{i} \mathbf{d}_{i}
$$

Note that the $\mathbf{x}_{K(j)}$ might have never existed in memory during the execution of the algorithm. Nevertheless, the iteration can be written as

$$
\begin{aligned}
\gamma_{j} & =\left(\mathbf{x}^{\star}-\mathbf{x}_{K(j)}, \mathbf{d}_{j}\right)_{\mathbf{A}} \\
\mathbf{x}_{j+1} & =\mathbf{x}_{j}+\beta \gamma_{j} \mathbf{d}_{j} .
\end{aligned}
$$


Obviously, enforcing consistent reads involves some overhead. In the analysis we consider both models, as the bounds for the inconsistent read model are not as good as the ones obtained when assuming consistent reads. There is clearly a trade-off here, which we present but do not attempt to quantify. It is a complex trade-off that depends on many factors, including possible hardware features like transactional memory that may enable efficient enforcement of consistent reads.

More importantly, in many cases even without any special provisions, the probability of an inconsistent read in an iteration is extremely small, so much that we do not expect it to happen much (or at all) in a normal execution of the algorithm. For the definition of consistent read to be violated in a certain iteration there must be two distinct indices $l$ and $c$ for which all of the following conditions are met:

1. $\mathbf{A}_{r c} \neq 0$ and $\mathbf{A}_{r l} \neq 0$ ( $r$ is the index picked in line 4$)$.

2. Both $(\mathbf{x})_{c}$ and $(\mathbf{x})_{l}$ are modified by other processors during the execution of line 5 (the read operation).

3. $(\mathbf{x})_{c}$ is read before $(\mathbf{x})_{l}$.

4. $(\mathbf{x})_{c}$ is modified (by another processor) after it is read, and $(\mathbf{x})_{l}$ is modified (by another processor) before it is read.

Having all these condition occur at the same time is rather rare. In fact, just having the first two occur is rather rare in the reference scenario. The reason is that each iteration reads at most $C_{2} \ll n$ entries. Suppose there are $u$ updates while reading those entries. Each such update affects a single random entry. Therefore, the probability that it will update one of the $C_{2}$ entries being read is at most $C_{2} / n$. The probability of getting two such updates is bounded by the probability of getting at least two in a binomial distribution with $u$ experiments and probability $C_{2} / n$. Unless $u$ is very large, this is an extremely small probability (since $C_{2} / n$ is tiny).

The discussion above suggests that in many cases the bound we obtain for the consistent read will be rather descriptive even if no special provisions are taken to enforce the consistent read assumption. That is, we expect the actual behavior to be somewhere between the bound for the consistent read and that for the inconsistent read, but closer to the one for consistent read.

We are mainly interested in algorithms with provable convergence rate. In a totally asynchronous model with arbitrary delays, there can also be an arbitrary delay in convergence. Therefore, we assume that asynchronism is bounded in the sense that delays are bounded.

Assumption A-3 (Bounded Asynchronism). There is a constant $\tau$ (measure of asynchronism) such that all updates that are older than $\tau$ iterations participate in the computation of iteration $j$, for all iterations $j=1,2, \ldots$.

In the consistent read model, this assumption translates to requiring that

$$
j-\tau \leq k(j) \leq j .
$$

In the inconsistent read model, this assumption translates to requiring that

$$
\{0,1, \ldots, \max \{0, j-\tau-1\}\} \subseteq K(j) .
$$

Since the running time of an iteration is proportional to the number of non-zeros in the row, a reasonable upper bound on $\tau$ is $c \cdot C_{2} \cdot P / C_{1}$ for some small constant $c$. However, this is probably a pessimistic upper bound, and in general when the variance in the number of non-zeros per row is not too large relative to the mean, we expect $\tau$ to be of order of $P$. Regardless, it is clear that in the reference scenario $\tau=O(P)$ (recall that we assume that $C_{2} / C_{1}$ is a small constant).

We now discuss the relation between $k(0), k(1), \ldots$ or $K(0), K(1), \ldots$ and the random variables $\mathbf{d}_{0}, \mathbf{d}_{1}, \ldots$ If we inspect the pseudo-code of Algorithm 1 closely we will realize that $k(j)$ or $K(j)$ (depending on the model) depend on the random choices $\mathbf{d}_{0}, \mathbf{d}_{1}, \ldots, \mathbf{d}_{j-1}$ made before the write operation, and more crucially on the random choice $\mathbf{d}_{j}$. The reason is that on line 5 we read only the relevant entries of $\mathbf{x}$, so only a small set of updates can be considered for 
inclusion. The set of relevant entries is determined by the selection of $\mathbf{d}_{j}$. However, a completely adversarial model which allows dependence of $k(j)$ ( or $K(j)$ ) on $\mathbf{d}_{0}, \mathbf{d}_{1}, \ldots, \mathbf{d}_{j}$ (for $j=1,2, \ldots$ ) and analyzes the worst-case behavior is not likely to be very faithful to the actual behavior of the algorithm. Therefore, we assume the delays are independent of the random choices, but allow them to be arbitrary (as long as the bounded asynchronism assumption holds). We acknowledge that this assumption cannot be enforced without paying a significant penalty in terms of iteration costs (e.g., the assumption is satisfied if the algorithm reads all the entries of $\mathbf{x}$ in each step)

Assumption A-4 (Independent Delays). We allow an arbitrary set of delays that satisfy (6) or (7) (depending on the context), but they do not depend on the random choices $\mathbf{d}_{0}, \mathbf{d}_{1}, \ldots$.

Finally, we remark on the role of the various assumptions in the analysis. Assumptions A-1 and A-2 allow us to write well defined iterations (iterations (4) and (5)) that can be analyzed mathematically. Assumption A-3 allows us to bound the number of elements in $\mathbf{x}_{j}-\mathbf{x}_{k(j)}\left(\right.$ or $\left.\mathbf{x}_{j}-\mathbf{x}_{K(j)}\right)$ that are non-zero by $\tau$, therefore implying that $\left(\mathbf{x}^{\star}-\right.$ $\left.\mathbf{x}_{k(j)}, \mathbf{d}_{j}\right)_{\mathbf{A}}=\left(\mathbf{x}^{\star}-\mathbf{x}_{j}, \mathbf{d}_{j}\right)_{\mathbf{A}}$ (respectively, $\left.\left(\mathbf{x}^{\star}-\mathbf{x}_{K(j)}, \mathbf{d}_{j}\right)_{\mathbf{A}}=\left(\mathbf{x}^{\star}-\mathbf{x}_{j}, \mathbf{d}_{j}\right)_{\mathbf{A}}\right)$ with high probability (as long as $\tau$ is small relative to $n$ ), which implies that the computed step-size is correct in most iterations. Assumption A-4 allows us to treat $k(j)$ and $K(j)$, in the proofs, as deterministic even though they might be random. Since $k(0), k(1), \ldots$ (or $K(0), K(1), \ldots$ ) do not depend on $\mathbf{d}_{0}, \mathbf{d}_{1}, \ldots$ we can condition on choices for which equation (6) (respectively, equation (7) holds, and any bound that does not depend on $k(0), k(1), \ldots$ (respectively, $K(0), K(1), \ldots)$ will hold for random ones as well.

For clarity, we now detail explicitly the two models we analyze in this paper.

Consistent Read Model. Algorithm 1 executed on all processors using the same x (i.e., all processors read and update the same $\mathbf{x}$ stored in a shared memory) with all four assumptions (A-1 to A-4). The governing iteration is:

$$
\begin{aligned}
j-\tau & \leq k(j) \leq j \\
\mathbf{d}_{j} & \sim U\left(\mathbf{e}^{(1)}, \ldots, \mathbf{e}^{(n)}\right) \\
\gamma_{j} & =\left(\mathbf{x}^{\star}-\mathbf{x}_{k(j)}, \mathbf{d}_{j}\right)_{\mathbf{A}} \\
\mathbf{x}_{j+1} & =\mathbf{x}_{j}+\beta \gamma_{j} \mathbf{d}_{j}
\end{aligned}
$$

with the additional assumptions that $\mathbf{d}_{0}, \mathbf{d}_{1}, \ldots$ are i.i.d, and that $k(0), k(1), \ldots$ do not depend on the random choices $\mathbf{d}_{0}, \mathbf{d}_{1}, \ldots$. In the above, $U\left(\mathbf{e}^{(1)}, \ldots, \mathbf{e}^{(n)}\right)$ denotes a uniform distribution on the $n$-dimensional identity vectors.

Inconsistent Read Model. Algorithm 1 executed on all processors using the same $\mathrm{x}$ (i.e., all processors read and update the same $\mathbf{x}$ stored in a shared memory) with assumptions A-1, A-3 and A-4. The governing iteration is:

$$
\begin{aligned}
&\{0, \ldots, \max \{0, j-\tau-1\}\} \subseteq K(j) \subseteq\{0, \ldots, j\} \\
& \mathbf{d}_{j} \sim U\left(\mathbf{e}^{(1)}, \ldots, \mathbf{e}^{(n)}\right) \\
& \gamma_{j}=\left(\mathbf{x}^{\star}-\mathbf{x}_{K(j)}, \mathbf{d}_{j}\right)_{\mathbf{A}} \\
& \mathbf{x}_{j+1}=\mathbf{x}_{j}+\beta \gamma_{j} \mathbf{d}_{j}
\end{aligned}
$$

with the additional assumptions that $\mathbf{d}_{0}, \mathbf{d}_{1}, \ldots$ are i.i.d, and that $K(0), K(1), \ldots$ do not depend on the random choices $\mathbf{d}_{0}, \mathbf{d}_{1}, \ldots$

\section{Convergence Bound with Consistent Read and Unit Step-size $(\beta=1)$}

In this section, we analyze the iteration under the consistent read model, i.e. iteration (8). For the moment, we consider only unit step-size $(\beta=1)$. 
Theorem 2. Consider iteration (8) with $\beta=1$ for an arbitrary starting vector $\mathbf{x}_{0}$, that is iteration (4) where $\mathbf{d}_{0}, \mathbf{d}_{1}, \ldots$ are i.i.d. vectors that take $\mathbf{e}^{(1)}, \ldots, \mathbf{e}^{(n)}$ with equal probability, and $k(0), k(1), \ldots$ are such that $(6)$ holds but are independent of the random choices of $\mathbf{d}_{0}, \mathbf{d}_{1}, \ldots$ Let $\rho=\frac{1}{n}\|\mathbf{A}\|_{\infty}=\max _{l}\left\{\frac{1}{n} \sum_{r=1}^{n}\left|\mathbf{A}_{l r}\right|\right\}$. Provided that $2 \rho \tau<1$, the following holds:

(a) For every $m \geq \frac{\log (1 / 2)}{\log \left(1-\lambda_{\max } / n\right)} \approx \frac{0.693 n}{\lambda_{\max }}$ we have

$$
E_{m} \leq\left(1-\frac{\nu_{\tau}}{2 \kappa}\right) E_{0}
$$

where

$$
\nu_{\tau}=1-2 \rho \tau
$$

(b) Let $T_{0}=\left\lceil\frac{\log (1 / 2)}{\log \left(1-\lambda_{\max } / n\right)}\right\rceil$ and $T=T_{0}+\tau$. For every $m \geq r T(r=1,2, \ldots)$ we have

$$
E_{m} \leq\left(1-\frac{\nu_{\tau}}{2 \kappa}\right)\left(1-\frac{\nu_{\tau}\left(1-\lambda_{\max } / n\right)^{\tau}}{2 \kappa}+\chi\right)^{r-1} E_{0}
$$

where

$$
\chi=\frac{\rho \tau^{2} \lambda_{\max }\left(1-\lambda_{\max } / n\right)^{-2 \tau}}{n} .
$$

Proof. In the proof, we use the following abbreviations:

$$
\delta_{\min }=\frac{\nu_{\tau} \lambda_{\min }}{n}, \quad \delta_{\max }=1-\frac{\lambda_{\max }}{n} .
$$

We begin with simple algebraic manipulations:

$$
\begin{aligned}
\left\|\mathbf{x}_{j+1}-\mathbf{x}^{\star}\right\|_{\mathbf{A}}^{2}= & \left\|\mathbf{x}_{j}+\gamma_{j} \mathbf{d}_{j}-\mathbf{x}^{\star}\right\|_{\mathbf{A}}^{2} \\
= & \left\|\mathbf{x}_{j}-\mathbf{x}^{\star}\right\|_{\mathbf{A}}^{2}+\left\|\gamma_{j} \mathbf{d}_{j}\right\|_{\mathbf{A}}^{2}+2\left(\mathbf{x}_{j}-\mathbf{x}^{\star}, \gamma_{j} \mathbf{d}_{j}\right)_{\mathbf{A}} \\
= & \left\|\mathbf{x}_{j}-\mathbf{x}^{\star}\right\|_{\mathbf{A}}^{2}+\gamma_{j}^{2}+2 \gamma_{j}\left(\mathbf{x}_{j}-\mathbf{x}^{\star}, \mathbf{d}_{j}\right)_{\mathbf{A}} \\
= & \left\|\mathbf{x}_{j}-\mathbf{x}^{\star}\right\|_{\mathbf{A}}^{2}+\left(\mathbf{x}_{k(j)}-\mathbf{x}^{\star}, \mathbf{d}_{j}\right)_{\mathbf{A}}^{2}-2\left(\mathbf{x}_{k(j)}-\mathbf{x}^{\star}, \mathbf{d}_{j}\right)_{\mathbf{A}}\left(\mathbf{x}_{j}-\mathbf{x}^{\star}, \mathbf{d}_{j}\right)_{\mathbf{A}} \\
= & \left\|\mathbf{x}_{j}-\mathbf{x}^{\star}\right\|_{\mathbf{A}}^{2}+\left(\mathbf{x}_{k(j)}-\mathbf{x}^{\star}, \mathbf{d}_{j}\right)_{\mathbf{A}}^{2} \\
& \quad-2\left(\mathbf{x}_{k(j)}-\mathbf{x}^{\star}, \mathbf{d}_{j}\right)_{\mathbf{A}}\left[\left(\mathbf{x}_{j}-\mathbf{x}_{k(j)}, \mathbf{d}_{j}\right)_{\mathbf{A}}+\left(\mathbf{x}_{k(j)}-\mathbf{x}^{\star}, \mathbf{d}_{j}\right)_{\mathbf{A}}\right] \\
= & \left\|\mathbf{x}_{j}-\mathbf{x}^{\star}\right\|_{\mathbf{A}}^{2}-\left(\mathbf{x}_{k(j)}-\mathbf{x}^{\star}, \mathbf{d}_{j}\right)_{\mathbf{A}}^{2}-2\left(\mathbf{x}_{k(j)}-\mathbf{x}^{\star}, \mathbf{d}_{j}\right)_{\mathbf{A}}\left(\mathbf{x}_{j}-\mathbf{x}_{k(j)}, \mathbf{d}_{j}\right)_{\mathbf{A}}
\end{aligned}
$$

In the above we use the fact that $\mathbf{A}$ has unit diagonal, so $\left(\mathbf{d}_{i}, \mathbf{d}_{i}\right)_{\mathbf{A}}=1$ for all $i$. We see that the error decreases by a positive "progress term" $\left(\left(\mathbf{x}_{k(j)}-\mathbf{x}^{\star}, \mathbf{d}_{j}\right)_{\mathbf{A}}^{2}\right)$, and it changes by an additional term $\left(2\left(\mathbf{x}_{k(j)}-\mathbf{x}^{\star}, \mathbf{d}_{j}\right)_{\mathbf{A}}\left(\mathbf{x}_{k(j)}-\right.\right.$ $\left.\left.\mathbf{x}_{j}, \mathbf{d}_{j}\right)_{\mathbf{A}}\right)$, which might be positive or negative. When the iterations are synchronized $(k(j)=j)$, there is no additional term, and the analysis reduces to the analysis of synchronous randomized Gauss-Seidel.

We first bound the additional term:

$$
\begin{aligned}
2\left(\mathbf{x}_{k(j)}-\mathbf{x}^{\star}, \mathbf{d}_{j}\right)_{\mathbf{A}}\left(\mathbf{x}_{j}-\mathbf{x}_{k(j)}, \mathbf{d}_{j}\right)_{\mathbf{A}} & =2\left(\mathbf{x}_{k(j)}-\mathbf{x}^{\star}, \mathbf{d}_{j}\right)_{\mathbf{A}}\left(\sum_{t=k(j)}^{j-1} \gamma_{t} \mathbf{d}_{t}, \mathbf{d}_{j}\right)_{\mathbf{A}} \\
& =\sum_{t=k(j)}^{j-1} 2\left(\mathbf{x}_{k(j)}-\mathbf{x}^{\star}, \mathbf{d}_{j}\right)_{\mathbf{A}}\left(\mathbf{x}^{\star}-\mathbf{x}_{k(t)}, \mathbf{d}_{t}\right)_{\mathbf{A}}\left(\mathbf{d}_{t}, \mathbf{d}_{j}\right)_{\mathbf{A}} \\
& \geq-\sum_{t=k(j)}^{j-1}\left[\left(\mathbf{x}_{k(j)}-\mathbf{x}^{\star}, \mathbf{d}_{j}\right)_{\mathbf{A}}^{2}\left|\left(\mathbf{d}_{t}, \mathbf{d}_{j}\right)_{\mathbf{A}}\right|+\left(\mathbf{x}_{k(t)}-\mathbf{x}^{\star}, \mathbf{d}_{t}\right)_{\mathbf{A}}^{2}\left|\left(\mathbf{d}_{t}, \mathbf{d}_{j}\right)_{\mathbf{A}}\right|\right] .
\end{aligned}
$$


Since $k(j) \leq t<j$ :

$$
\begin{aligned}
\mathbb{E}\left[\left(\mathbf{x}_{k(j)}-\mathbf{x}^{\star}, \mathbf{d}_{j}\right)_{\mathbf{A}}^{2}\left|\left(\mathbf{d}_{t}, \mathbf{d}_{j}\right)_{\mathbf{A}}\right|\right] & =\mathbb{E}\left[\mathbb{E}\left[\left(\mathbf{x}_{k(j)}-\mathbf{x}^{\star}, \mathbf{d}_{j}\right)_{\mathbf{A}}^{2}\left|\left(\mathbf{d}_{t}, \mathbf{d}_{j}\right)_{\mathbf{A}}\right| \mid \mathbf{d}_{0}, \ldots, \mathbf{d}_{t-1}\right]\right] \\
& =\mathbb{E}\left[\frac{1}{n^{2}} \sum_{l=1}^{n} \sum_{r=1}^{n}\left(\mathbf{x}_{k(j)}-\mathbf{x}^{\star}, \mathbf{e}^{(l)}\right)_{\mathbf{A}}^{2}\left|\left(\mathbf{e}^{(l)}, \mathbf{e}^{(r)}\right)_{\mathbf{A}}\right|\right] \\
& =\mathbb{E}\left[\frac{1}{n^{2}} \sum_{l=1}^{n} \sum_{r=1}^{n}\left(\mathbf{x}_{k(j)}-\mathbf{x}^{\star}, \mathbf{e}^{(l)}\right)_{\mathbf{A}}^{2}\left|\mathbf{A}_{l r}\right|\right] \\
& \leq \rho \mathbb{E}\left[\frac{1}{n} \sum_{l=1}^{n}\left(\mathbf{x}_{k(j)}-\mathbf{x}^{\star}, \mathbf{e}^{(l)}\right)_{\mathbf{A}}^{2}\right]=\rho \mathbb{E}\left[\left(\mathbf{x}_{k(j)}-\mathbf{x}^{\star}, \mathbf{d}_{j}\right)_{\mathbf{A}}^{2}\right] .
\end{aligned}
$$

Similarly, $\mathbb{E}\left[\left(\mathbf{x}_{k(t)}-\mathbf{x}^{\star}, \mathbf{d}_{t}\right)_{\mathbf{A}}^{2}\left|\left(\mathbf{d}_{t}, \mathbf{d}_{j}\right)_{\mathbf{A}}\right|\right] \leq \rho \mathbb{E}\left[\left(\mathbf{x}_{k(t)}-\mathbf{x}^{\star}, \mathbf{d}_{t}\right)_{\mathbf{A}}^{2}\right]$. Taking expectation of (11) and applying the last inequality we find that

$$
\begin{aligned}
\mathbb{E}\left[2\left(\mathbf{x}_{k(j)}-\mathbf{x}^{\star}, \mathbf{d}_{j}\right)_{\mathbf{A}}\left(\mathbf{x}_{j}-\mathbf{x}_{k(j)}, \mathbf{d}_{j}\right)_{\mathbf{A}}\right] & \geq-\rho \sum_{t=k(j)}^{j-1}\left[\mathbb{E}\left[\left(\mathbf{x}_{k(j)}-\mathbf{x}^{\star}, \mathbf{d}_{j}\right)_{\mathbf{A}}^{2}\right]+\mathbb{E}\left[\left(\mathbf{x}_{k(t)}-\mathbf{x}^{\star}, \mathbf{d}_{t}\right)_{\mathbf{A}}^{2}\right]\right] \\
& =-\rho|j-k(j)| \mathbb{E}\left[\left(\mathbf{x}_{k(j)}-\mathbf{x}^{\star}, \mathbf{d}_{j}\right)_{\mathbf{A}}^{2}\right]-\rho \sum_{t=k(j)}^{j-1} \mathbb{E}\left[\left(\mathbf{x}_{k(t)}-\mathbf{x}^{\star}, \mathbf{d}_{t}\right)_{\mathbf{A}}^{2}\right] \\
& \geq-\rho \tau \mathbb{E}\left[\left(\mathbf{x}_{k(j)}-\mathbf{x}^{\star}, \mathbf{d}_{j}\right)_{\mathbf{A}}^{2}\right]-\rho \sum_{t=k(j)}^{j-1} \mathbb{E}\left[\left(\mathbf{x}_{k(t)}-\mathbf{x}^{\star}, \mathbf{d}_{t}\right)_{\mathbf{A}}^{2}\right] .
\end{aligned}
$$

Taking expectation of (10), and plugging in the last inequality, we find that

$$
E_{j+1} \leq E_{j}-(1-\rho \tau) \mathbb{E}\left[\left(\mathbf{x}_{k(j)}-\mathbf{x}^{\star}, \mathbf{d}_{j}\right)_{\mathbf{A}}^{2}\right]+\rho \sum_{t=k(j)}^{j-1} \mathbb{E}\left[\left(\mathbf{x}_{k(t)}-\mathbf{x}^{\star}, \mathbf{d}_{t}\right)_{\mathbf{A}}^{2}\right] .
$$

Unrolling the recursion, we find that for every $m$ :

$$
E_{m} \leq E_{0}-(1-\rho \tau) \sum_{i=0}^{m-1} \mathbb{E}\left[\left(\mathbf{x}_{k(i)}-\mathbf{x}^{\star}, \mathbf{d}_{i}\right)_{\mathbf{A}}^{2}\right]+\rho \sum_{i=0}^{m-1} \sum_{t=k(i)}^{i-1} \mathbb{E}\left[\left(\mathbf{x}_{k(t)}-\mathbf{x}^{\star}, \mathbf{d}_{t}\right)_{\mathbf{A}}^{2}\right]
$$

In the last sum of the previous inequality $\left(\rho \sum_{i=0}^{m-1} \sum_{t=k(i)}^{i-1} \mathbb{E}\left[\left(\mathbf{x}_{k(t)}-\mathbf{x}^{\star}, \mathbf{d}_{t}\right)_{\mathbf{A}}^{2}\right]\right)$, each term of the form $\mathbb{E}\left[\left(\mathbf{x}_{k(r)}-\mathbf{x}^{\star}, \mathbf{d}_{r}\right)_{\mathbf{A}}^{2}\right]$ appears at most $\tau$ times, each time with a coefficient $\rho$. So

$$
E_{m} \leq E_{0}-(1-2 \rho \tau) \sum_{i=0}^{m-1} \mathbb{E}\left[\left(\mathbf{x}_{k(i)}-\mathbf{x}^{\star}, \mathbf{d}_{i}\right)_{\mathbf{A}}^{2}\right] .
$$

We now apply the bound $\mathbb{E}\left[\left(\mathbf{x}_{k(i)}-\mathbf{x}^{\star}, \mathbf{d}_{i}\right)_{\mathbf{A}}^{2}\right] \geq\left(\lambda_{\min } / n\right) E_{k(i)}($ Lemma 1$)$, to find that

$$
E_{m} \leq E_{0}-\delta_{\min } \sum_{i=0}^{m-1} E_{k(i)}
$$

Proof of (a). Lemma 1 implies that for any $b \geq a$ we have $E_{b} \geq \delta_{\max }^{b-a} E_{a}$. Indeed, 


$$
\begin{aligned}
\left\|\mathbf{x}_{j+1}-\mathbf{x}^{\star}\right\|_{\mathbf{A}}^{2}= & \left\|\mathbf{x}_{j}+\gamma_{j} \mathbf{d}_{j}-\mathbf{x}^{\star}\right\|_{\mathbf{A}}^{2} \\
= & \left\|\mathbf{x}_{j}-\mathbf{x}^{\star}\right\|_{\mathbf{A}}^{2}+\gamma_{j}^{2}+2 \gamma_{j}\left(\mathbf{x}_{j}-\mathbf{x}^{\star}, \mathbf{d}_{j}\right)_{\mathbf{A}} \\
= & \left\|\mathbf{x}_{j}-\mathbf{x}^{\star}\right\|_{\mathbf{A}}^{2}+\left(\mathbf{x}_{k(j)}-\mathbf{x}^{\star}, \mathbf{d}_{j}\right)_{\mathbf{A}}^{2}-2\left(\mathbf{x}_{k(j)}-\mathbf{x}^{\star}, \mathbf{d}_{j}\right)_{\mathbf{A}}\left(\mathbf{x}_{j}-\mathbf{x}^{\star}, \mathbf{d}_{j}\right)_{\mathbf{A}} \\
= & \left\|\mathbf{x}_{j}-\mathbf{x}^{\star}\right\|_{\mathbf{A}}^{2}+\left(\mathbf{x}_{k(j)}-\mathbf{x}_{j}+\mathbf{x}_{j}-\mathbf{x}^{\star}, \mathbf{d}_{j}\right)_{\mathbf{A}}^{2}-2\left(\mathbf{x}_{k(j)}-\mathbf{x}^{\star}, \mathbf{d}_{j}\right)_{\mathbf{A}}\left(\mathbf{x}_{j}-\mathbf{x}^{\star}, \mathbf{d}_{j}\right)_{\mathbf{A}} \\
= & \left\|\mathbf{x}_{j}-\mathbf{x}^{\star}\right\|_{\mathbf{A}}^{2}+\left(\mathbf{x}_{k(j)}-\mathbf{x}^{\star}, \mathbf{d}_{j}\right)_{\mathbf{A}}^{2}+\left(\mathbf{x}_{k(j)}-\mathbf{x}_{j}, \mathbf{d}_{j}\right)_{\mathbf{A}}^{2} \\
& \quad+2\left(\mathbf{x}_{j}-\mathbf{x}^{\star}, \mathbf{d}_{j}\right)_{\mathbf{A}}\left(\mathbf{x}_{k(j)}-\mathbf{x}_{j}, \mathbf{d}_{j}\right)_{\mathbf{A}} \\
& \quad-2\left(\mathbf{x}_{k(j)}-\mathbf{x}^{\star}, \mathbf{d}_{j}\right)_{\mathbf{A}}\left(\mathbf{x}_{j}-\mathbf{x}^{\star}, \mathbf{d}_{j}\right)_{\mathbf{A}} \\
= & \left\|\mathbf{x}_{j}-\mathbf{x}^{\star}\right\|_{\mathbf{A}}^{2}+\left(\mathbf{x}_{j}-\mathbf{x}^{\star}, \mathbf{d}_{j}\right)_{\mathbf{A}}^{2}+\left(\mathbf{x}_{k(j)}-\mathbf{x}_{j}, \mathbf{d}_{j}\right)_{\mathbf{A}}^{2} \\
& \quad-2\left(\mathbf{x}_{j}-\mathbf{x}^{\star}, \mathbf{d}_{j}\right)_{\mathbf{A}}\left(\mathbf{x}_{j}-\mathbf{x}^{\star}, \mathbf{d}_{j}\right)_{\mathbf{A}} \\
= & \left\|\mathbf{x}_{j}-\mathbf{x}^{\star}\right\|_{\mathbf{A}}^{2}-\left(\mathbf{x}_{j}-\mathbf{x}^{\star}, \mathbf{d}_{j}\right)_{\mathbf{A}}^{2}+\left(\mathbf{x}_{k(j)}-\mathbf{x}_{j}, \mathbf{d}_{j}\right)_{\mathbf{A}}^{2} \\
\geq & \left\|\mathbf{x}_{j}-\mathbf{x}^{\star}\right\|_{\mathbf{A}}^{2}-\left(\mathbf{x}_{j}-\mathbf{x}^{\star}, \mathbf{d}_{j}\right)_{\mathbf{A}}^{2}
\end{aligned}
$$

so by taking expectation and applying Lemma 1 (notice that $\mathbf{x}_{k(j)}$ is independent of $\mathbf{d}_{j}$ ), we find that $E_{j+1} \geq \delta_{\max } E_{j}$. In particular since $i \geq k(i)$,

$$
E_{k(i)} \geq \delta_{\max }^{k(i)} E_{0} \geq \delta_{\max }^{i} E_{0} .
$$

Plugging (14) into (13) we get the following inequality, which leads immediately to assertion (a):

$$
E_{m} \leq\left(1-\delta_{\min } \sum_{i=0}^{m-1} \delta_{\max }^{i}\right) E_{0}=\left(1-\frac{\delta_{\min }\left(1-\delta_{\max }^{m}\right)}{1-\delta_{\max }}\right) E_{0}=\left(1-\nu_{\tau} \kappa^{-1}\left(1-\delta_{\max }^{m}\right)\right) E_{0} .
$$

Proof of $(\mathbf{b})$. Let

$$
C_{i}=\{r T+i-\tau \leq t \leq r T+i-1: t \geq r T\}
$$

and

$$
D_{i}=\{r T+i-\tau \leq t \leq r T+i-1: t<r T\} .
$$

Unrolling the recursion in equation (12) starting at $r T$, we find that for $r \geq 1$ and $w \geq 0$

$$
\begin{aligned}
E_{(r+1) T+w} \leq & E_{r T}-(1-\rho \tau) \sum_{i=0}^{T-1+w} \mathbb{E}\left[\left(\mathbf{x}_{k(r T+i)}-\mathbf{x}^{\star}, \mathbf{d}_{r T+i}\right)_{\mathbf{A}}^{2}\right]+\rho \sum_{i=0}^{T-1+w} \sum_{t=k(r T+i)}^{r T+i-1} \mathbb{E}\left[\left(\mathbf{x}_{k(t)}-\mathbf{x}^{\star}, \mathbf{d}_{t}\right)_{\mathbf{A}}^{2}\right] \\
\leq & E_{r T}-(1-\rho \tau) \sum_{i=0}^{T-1+w} \mathbb{E}\left[\left(\mathbf{x}_{k(r T+i)}-\mathbf{x}^{\star}, \mathbf{d}_{r T+i}\right)_{\mathbf{A}}^{2}\right]+\rho \sum_{i=0}^{T-1+w} \sum_{t=r T+i-\tau}^{r T+i-1} \mathbb{E}\left[\left(\mathbf{x}_{k(t)}-\mathbf{x}^{\star}, \mathbf{d}_{t}\right)_{\mathbf{A}}^{2}\right] \\
\leq & E_{r T}-(1-\rho \tau) \sum_{i=0}^{T-1+w} \mathbb{E}\left[\left(\mathbf{x}_{k(r T+i)}-\mathbf{x}^{\star}, \mathbf{d}_{r T+i}\right)_{\mathbf{A}}^{2}\right]+\rho \sum_{i=0}^{T-1+w} \sum_{t \in C_{i}} \mathbb{E}\left[\left(\mathbf{x}_{k(t)}-\mathbf{x}^{\star}, \mathbf{d}_{t}\right)_{\mathbf{A}}^{2}\right] \\
& \quad+\rho \sum_{i=0}^{T-1+w} \sum_{t \in D_{i}} \mathbb{E}\left[\left(\mathbf{x}_{k(t)}-\mathbf{x}^{\star}, \mathbf{d}_{t}\right)_{\mathbf{A}}^{2}\right] \\
\leq & E_{r T}-(1-2 \rho \tau) \sum_{i=0}^{T-1+w} \mathbb{E}\left[\left(\mathbf{x}_{k(r T+i)}-\mathbf{x}^{\star}, \mathbf{d}_{r T+i}\right)_{\mathbf{A}}^{2}\right]+\rho \sum_{i=0}^{\tau-1} \sum_{t \in D_{i}} \mathbb{E}\left[\left(\mathbf{x}_{k(t)}-\mathbf{x}^{\star}, \mathbf{d}_{t}\right)_{\mathbf{A}}^{2}\right] . \\
\leq & E_{r T}-(1-2 \rho \tau) \sum_{i=\tau}^{T-1+w} \mathbb{E}\left[\left(\mathbf{x}_{k(r T+i)}-\mathbf{x}^{\star}, \mathbf{d}_{r T+i}\right)_{\mathbf{A}}^{2}\right]+\rho \sum_{i=0}^{\tau-1} \sum_{t \in D_{i}} \mathbb{E}\left[\left(\mathbf{x}_{k(t)}-\mathbf{x}^{\star}, \mathbf{d}_{t}\right)_{\mathbf{A}}^{2}\right] . \quad(15)
\end{aligned}
$$


The second-to-last inequality follows from the fact that each term of the form $\mathbb{E}\left[\left(\mathbf{x}_{k(l)}-\mathbf{x}^{\star}, \mathbf{d}_{l}\right)_{\mathbf{A}}^{2}\right]$ appears at most $\tau$ times in $\rho \sum_{i=0}^{T-1+w} \sum_{t \in C_{i}} \mathbb{E}\left[\left(\mathbf{x}_{k(t)}-\mathbf{x}^{\star}, \mathbf{d}_{t}\right)_{\mathbf{A}}^{2}\right]$. We also use the fact that for $i \geq \tau$ we trivially have $D_{i}=\emptyset$. We first bound $E_{r T}-(1-2 \rho \tau) \sum_{i=\tau}^{T-1+w} \mathbb{E}\left[\left(\mathbf{x}_{k(r T+i)}-\mathbf{x}^{\star}, \mathbf{d}_{r T+i}\right)_{\mathbf{A}}^{2}\right]$. Using Lemma 1 .

$$
E_{r T}-(1-2 \rho \tau) \sum_{i=\tau}^{T-1+w} \mathbb{E}\left[\left(\mathbf{x}_{k(r T+i)}-\mathbf{x}^{\star}, \mathbf{d}_{r T+i}\right)_{\mathbf{A}}^{2}\right] \leq E_{r T}-\delta_{\min } \sum_{i=\tau}^{T-1+w} E_{k(r T+i)} .
$$

Since $i \geq \tau$ we have $k(k T+i) \geq k T$ so $E_{k(r T+i)} \geq \delta_{\max }^{k(r T+i)-r T} E_{r T} \geq \delta_{\max }^{i} E_{r T}$. Therefore

$$
E_{r T}-(1-2 \rho \tau) \sum_{i=\tau}^{T-1+w} \mathbb{E}\left[\left(\mathbf{x}_{k(r T+i)}-\mathbf{x}^{\star}, \mathbf{d}_{r T+i}\right)_{\mathbf{A}}^{2}\right] \leq\left(1-\delta_{\min } \delta_{\max }^{\tau} \sum_{i=0}^{T-1+w-\tau} \delta_{\max }^{i}\right) E_{r T} .
$$

Noticing that $T-1+w-\tau=T_{0}-1+w$ and bounding the geometric sum as in assertion (a), we find that $\left(1-\delta_{\min } \delta_{\max }^{\tau} \sum_{i=0}^{T-1+w-\tau} \delta_{\max }^{i}\right) \leq\left(1-\frac{\delta_{\max }^{\tau} \nu_{\tau}}{2 \kappa}\right)$, so

$$
E_{r T}-(1-2 \rho \tau) \sum_{i=\tau}^{T-1+w} \mathbb{E}\left[\left(\mathbf{x}_{k(r T+i)}-\mathbf{x}^{\star}, \mathbf{d}_{r T+i}\right)_{\mathbf{A}}^{2}\right] \leq\left(1-\frac{\delta_{\max }^{\tau} \nu_{\tau}}{2 \kappa}\right) E_{r T} .
$$

We now bound $\rho \sum_{i=0}^{\tau-1} \sum_{t \in D_{i}} \mathbb{E}\left[\left(\mathbf{x}_{k(t)}-\mathbf{x}^{\star}, \mathbf{d}_{t}\right)_{\mathbf{A}}^{2}\right]$. Recall that for every $b \geq a$ we have $E_{b} \geq \delta_{\max }^{b-a} E_{a}$, so, for $i=0, \ldots, \tau-1$ and $t \in D_{i}$ we have

$$
E_{k(t)} \leq \delta_{\max }^{k(t)-r T} E_{r T} \leq \delta_{\max }^{-2 \tau} E_{r T} .
$$

The last inequality follows from the fact that for $t \in D_{i}, k(t)-r T \geq-2 \tau$ and $\delta_{\max }<1$. We now bound

$$
\rho \sum_{i=0}^{\tau-1} \sum_{t \in D_{i}} \mathbb{E}\left[\left(\mathbf{x}_{k(t)}-\mathbf{x}^{\star}, \mathbf{d}_{t}\right)_{\mathbf{A}}^{2}\right] \leq \rho \sum_{i=0}^{\tau-1} \sum_{t \in D_{i}} \frac{\lambda_{\max } \delta_{\max }^{-2 \tau}}{n} E_{r T} \leq \frac{\rho \tau^{2} \lambda_{\max } \delta_{\max }^{-2 \tau}}{n} E_{r T}=\chi E_{r T} .
$$

Combine the last inequality with $(16)$ and assertion (a) to complete the proof of assertion (b).

\section{Discussion:}

- Assertion (a) shows that after we perform enough asynchronous iterations, we are guaranteed to reduce the expected error by a constant factor. In order to drive the expected error down to an arbitrary fraction of the input error, we can adopt the following scheme. We start with asynchronous iterations. After $n$ iterations have been completed we synchronize the threads and restart the iterations. The matrix $\mathbf{A}$ has unit diagonal, so $\lambda_{\max } \geq 1$. Therefore, by performing $k \geq n$ iterations, we are guaranteeing a $1-\nu_{\tau} / 2 \kappa$ factor reduction in the expected error. We then continue to iterate and synchronize until the expected error is guaranteed to be small enough. The number of outer iterations until convergence (reduction of error by a predetermined factor) is $O\left(\kappa / \nu_{\tau}\right)$. This is also the number of synchronization points. When $\nu_{\tau}$ is close to one, the number of synchronization points is asymptotically the same as in Jacobi, but the convergence rate is that of Gauss-Seidel. Furthermore, we do not need to really divide the iterations between processors (basically, every processor can do as many iterations as it can, until synchronization) and it is not important to synchronize exactly after $n$ iterations. So, from a practical perspective, a time based scheme for synchronizing the processors should be sufficient, and will not suffer from large wait times due to load imbalance.

- Assertion (b) shows that even if we do not occasionally synchronize the threads, we still get long-term linear convergence, but at a slower rate. We say convergence is linear in the long term since we cannot guarantee a diminishing bound in every iteration, but we can prove a constant factor reduction over a large enough number of iterations. 
- The terms $\delta_{\max }^{\tau}$ and $\delta_{\max }^{-2 \tau}$ that appear in assertion (b) might seem problematic as they are exponential in the number of processors (because $\tau=\Omega(P)$ ). However, in our reference scenario this is not an issue because $\lambda_{\max }=O(1)$ and $\tau \ll n$ (since we assume that $P \ll n$ ), so $\delta_{\max }^{\tau}$ and $\delta_{\max }^{-2 \tau}$ are actually very close to 1 .

- The number of iterations to guarantee a $1-\nu_{\tau} / 2 \kappa$ reduction of expected error (as in assertion (a)) in synchronous randomized Gauss-Seidel is approximately $\nu_{\tau} n / 2 \lambda_{\max }$. When $\nu_{\tau}$ is close to one this is only slightly better than the bound for AsyRGS, which is a small price to pay for the good speedups expected for the asynchronous algorithm.

- Consider our reference scenario in a weak-scaling regime (i.e., $P \approx c n$ for a very small $c$ ). In this case, $\nu_{\tau}$ is bounded by a constant close to one because $\rho=O(1 / n)$ in the reference scenario. Therefore, with occasional synchronization of the threads, the number of iterations increases by a small constant factor due to asynchronism. That is, the asynchronous phases do not violate the weak-scaling, although the number of iterations can increase due to $\lambda_{\min }$ becoming smaller. As for the case where only asynchronous iterations are used, we have $\chi \approx c^{2} \lambda_{\max }^{2}$. So, $\chi$ itself exhibits weak scaling. However, its value should be interpreted with respect to $\kappa^{-1}$. If $\lambda_{\min }$ shrinks as $n$ grows, as is the case in many applications, then the relative size of $\chi$ grows and we do not have weak scaling.

- In general, if $\rho=O(1 / n)$, we have $\nu_{\tau}=O(1)$ and the discussion in the previous paragraph applies. Sparsity is not the only scenario in which $\rho=O(1 / n)$ : for example, $\rho \leq 2 / n$ if $\mathbf{A}$ is symmetric diagonally dominant, regardless of sparsity. Other strong decay properties of off-diagonal entries might guarantee $\rho=O(1 / n)$ as well.

\section{Improving Scalability by Controlling Step-Size}

If we inspect (2) we see that the best bound is attained for unit step-size. Griebel and Oswald introduced a step-size since it is known that for certain applications over/under relaxations converge faster [9]. In this section, we show that by controlling the step-size, it is possible to have a convergent method for any delay (as long as we set the step size small enough), unlike the bound in Theorem 2 which requires $2 \rho \tau<1$. In addition, we show that by optimizing the step-size we can also improve the scaling (dependence on $\tau$ ) in our bounds.

Our more general analysis starts with some simple algebraic manipulations:

$$
\begin{aligned}
\left\|\mathbf{x}_{j+1}-\mathbf{x}^{\star}\right\|_{\mathbf{A}}^{2}= & \left\|\mathbf{x}_{j}+\beta \gamma_{j} \mathbf{d}_{j}-\mathbf{x}^{\star}\right\|_{\mathbf{A}}^{2} \\
= & \left\|\mathbf{x}_{j}-\mathbf{x}^{\star}\right\|_{\mathbf{A}}^{2}+\left\|\beta \gamma_{j} \mathbf{d}_{j}\right\|_{\mathbf{A}}^{2}+2\left(\mathbf{x}_{j}-\mathbf{x}^{\star}, \beta \gamma_{j} \mathbf{d}_{j}\right)_{\mathbf{A}} \\
= & \left\|\mathbf{x}_{j}-\mathbf{x}^{\star}\right\|_{\mathbf{A}}^{2}+\beta^{2} \gamma_{j}^{2}+2 \beta \gamma_{j}\left(\mathbf{x}_{j}-\mathbf{x}^{\star}, \mathbf{d}_{j}\right)_{\mathbf{A}} \\
= & \left\|\mathbf{x}_{j}-\mathbf{x}^{\star}\right\|_{\mathbf{A}}^{2}+\beta^{2}\left(\mathbf{x}_{k(j)}-\mathbf{x}^{\star}, \mathbf{d}_{j}\right)_{\mathbf{A}}^{2}-2 \beta\left(\mathbf{x}_{k(j)}-\mathbf{x}^{\star}, \mathbf{d}_{j}\right)_{\mathbf{A}}\left(\mathbf{x}_{j}-\mathbf{x}^{\star}, \mathbf{d}_{j}\right)_{\mathbf{A}} \\
= & \left\|\mathbf{x}_{j}-\mathbf{x}^{\star}\right\|_{\mathbf{A}}^{2}+\beta^{2}\left(\mathbf{x}_{k(j)}-\mathbf{x}^{\star}, \mathbf{d}_{j}\right)_{\mathbf{A}}^{2} \\
& \quad-2 \beta\left(\mathbf{x}_{k(j)}-\mathbf{x}^{\star}, \mathbf{d}_{j}\right)_{\mathbf{A}}\left[\left(\mathbf{x}_{j}-\mathbf{x}_{k(j)}, \mathbf{d}_{j}\right)_{\mathbf{A}}+\left(\mathbf{x}_{k(j)}-\mathbf{x}^{\star}, \mathbf{d}_{j}\right)_{\mathbf{A}}\right] \\
= & \left\|\mathbf{x}_{j}-\mathbf{x}^{\star}\right\|_{\mathbf{A}}^{2}-\beta(2-\beta)\left(\mathbf{x}_{k(j)}-\mathbf{x}^{\star}, \mathbf{d}_{j}\right)_{\mathbf{A}}^{2}-2 \beta\left(\mathbf{x}_{k(j)}-\mathbf{x}^{\star}, \mathbf{d}_{j}\right)_{\mathbf{A}}\left(\mathbf{x}_{j}-\mathbf{x}_{k(j)}, \mathbf{d}_{j}\right)_{\mathbf{A}}
\end{aligned}
$$

As before, we continue with bounding the additional term.

$$
\begin{aligned}
2 \beta\left(\mathbf{x}_{k(j)}-\mathbf{x}^{\star}, \mathbf{d}_{j}\right)_{\mathbf{A}}\left(\mathbf{x}_{j}-\mathbf{x}_{k(j)}, \mathbf{d}_{j}\right)_{\mathbf{A}} & =2 \beta\left(\mathbf{x}_{k(j)}-\mathbf{x}^{\star}, \mathbf{d}_{j}\right)_{\mathbf{A}}\left(\sum_{t=k(j)}^{j-1} \beta \gamma_{t} \mathbf{d}_{t}, \mathbf{d}_{j}\right)_{\mathbf{A}} \\
& =\beta^{2} \sum_{t=k(j)}^{j-1} 2\left(\mathbf{x}_{k(j)}-\mathbf{x}^{\star}, \mathbf{d}_{j}\right)_{\mathbf{A}}\left(\mathbf{x}^{\star}-\mathbf{x}_{k(t)}, \mathbf{d}_{t}\right)_{\mathbf{A}}\left(\mathbf{d}_{t}, \mathbf{d}_{j}\right)_{\mathbf{A}}
\end{aligned}
$$




$$
\geq-\beta^{2} \sum_{t=k(j)}^{j-1}\left[\left(\mathbf{x}_{k(j)}-\mathbf{x}^{\star}, \mathbf{d}_{j}\right)_{\mathbf{A}}^{2}\left|\left(\mathbf{d}_{t}, \mathbf{d}_{j}\right)_{\mathbf{A}}\right|+\left(\mathbf{x}_{k(t)}-\mathbf{x}^{\star}, \mathbf{d}_{t}\right)_{\mathbf{A}}^{2}\left|\left(\mathbf{d}_{t}, \mathbf{d}_{j}\right)_{\mathbf{A}}\right|\right] .
$$

We see that the progress term is $O(\beta)$, but the additional term is $O\left(\beta^{2}\right)$. In synchronous randomized Gauss-Seidel the best bound on the expected error is achieved with $\beta=1$, but for an asynchronous computation the best bound is achieved with some $\beta<1$ (depending on $\tau$ ).

It is still the case that $E_{j+1} \geq \delta_{\max } E_{j}$. Indeed,

$$
\begin{aligned}
\mathbf{x}_{j+1} & =\mathbf{x}_{j}+\beta \gamma_{j} \mathbf{d}_{j} \\
& =\mathbf{x}_{j}+\beta\left(\mathbf{x}^{\star}-\mathbf{x}_{k(j)}, \mathbf{d}_{j}\right)_{\mathbf{A}} \mathbf{d}_{j} \\
& =\mathbf{x}_{j}+\left(\beta \mathbf{x}^{\star}-\beta \mathbf{x}_{k(j)}, \mathbf{d}_{j}\right)_{\mathbf{A}} \mathbf{d}_{j} \\
& =\mathbf{x}_{j}+\left(\mathbf{x}^{\star}-\mathbf{y}, \mathbf{d}_{j}\right)_{\mathbf{A}} \mathbf{d}_{j}
\end{aligned}
$$

where $\mathbf{y}=(1-\beta) \mathbf{x}^{\star}+\mathbf{x}_{k(j)}$. Denote $\tilde{\gamma}_{j}=\left(\mathbf{x}^{\star}-\mathbf{y}, \mathbf{d}_{j}\right)_{\mathbf{A}}$. Now,

$$
\begin{aligned}
\left\|\mathbf{x}_{j+1}-\mathbf{x}^{\star}\right\|_{\mathbf{A}}^{2}= & \left\|\mathbf{x}_{j}+\tilde{\gamma}_{j} \mathbf{d}_{j}-\mathbf{x}^{\star}\right\|_{\mathbf{A}}^{2} \\
= & \left\|\mathbf{x}_{j}-\mathbf{x}^{\star}\right\|_{\mathbf{A}}^{2}+\left\|\tilde{\gamma}_{j} \mathbf{d}_{j}\right\|_{\mathbf{A}}^{2}+2\left(\mathbf{x}_{j}-\mathbf{x}^{\star}, \tilde{\gamma}_{j} \mathbf{d}_{j}\right)_{\mathbf{A}} \\
= & \left\|\mathbf{x}_{j}-\mathbf{x}^{\star}\right\|_{\mathbf{A}}^{2}+\tilde{\gamma}_{j}^{2}+2 \tilde{\gamma}_{j}\left(\mathbf{x}_{j}-\mathbf{x}^{\star}, \mathbf{d}_{j}\right)_{\mathbf{A}} \\
= & \left\|\mathbf{x}_{j}-\mathbf{x}^{\star}\right\|_{\mathbf{A}}^{2}+\left(\mathbf{y}-\mathbf{x}^{\star}, \mathbf{d}_{j}\right)_{\mathbf{A}}^{2}-2\left(\mathbf{y}-\mathbf{x}^{\star}, \mathbf{d}_{j}\right)_{\mathbf{A}}\left(\mathbf{x}_{j}-\mathbf{x}^{\star}, \mathbf{d}_{j}\right)_{\mathbf{A}} \\
= & \left\|\mathbf{x}_{j}-\mathbf{x}^{\star}\right\|_{\mathbf{A}}^{2}+\left(\mathbf{y}-\mathbf{x}_{j}+\mathbf{x}_{j}-\mathbf{x}^{\star}, \mathbf{d}_{j}\right)_{\mathbf{A}}^{2}-2\left(\mathbf{y}-\mathbf{x}^{\star}, \mathbf{d}_{j}\right)_{\mathbf{A}}\left(\mathbf{x}_{j}-\mathbf{x}^{\star}, \mathbf{d}_{j}\right)_{\mathbf{A}} \\
= & \left\|\mathbf{x}_{j}-\mathbf{x}^{\star}\right\|_{\mathbf{A}}^{2}+\left(\mathbf{x}_{j}-\mathbf{x}^{\star}, \mathbf{d}_{j}\right)_{\mathbf{A}}^{2}+\left(\mathbf{y}-\mathbf{x}_{j}, \mathbf{d}_{j}\right)_{\mathbf{A}}^{2} \\
& \quad+2\left(\mathbf{x}_{j}-\mathbf{x}^{\star}, \mathbf{d}_{j}\right)_{\mathbf{A}}\left(\mathbf{y}-\mathbf{x}_{j}, \mathbf{d}_{j}\right)_{\mathbf{A}} \\
& \quad-2\left(\mathbf{y}-\mathbf{x}^{\star}, \mathbf{d}_{j}\right)_{\mathbf{A}}\left(\mathbf{x}_{j}-\mathbf{x}^{\star}, \mathbf{d}_{j}\right)_{\mathbf{A}} \\
= & \left\|\mathbf{x}_{j}-\mathbf{x}^{\star}\right\|_{\mathbf{A}}^{2}+\left(\mathbf{x}_{j}-\mathbf{x}^{\star}, \mathbf{d}_{j}\right)_{\mathbf{A}}^{2}+\left(\mathbf{y}-\mathbf{x}_{j}, \mathbf{d}_{j}\right)_{\mathbf{A}}^{2} \\
& \quad-2\left(\mathbf{x}_{j}-\mathbf{x}^{\star}, \mathbf{d}_{j}\right)_{\mathbf{A}}\left(\mathbf{x}_{j}-\mathbf{x}^{\star}, \mathbf{d}_{j}\right)_{\mathbf{A}} \\
= & \left\|\mathbf{x}_{j}-\mathbf{x}^{\star}\right\|_{\mathbf{A}}^{2}-\left(\mathbf{x}_{j}-\mathbf{x}^{\star}, \mathbf{d}_{j}\right)_{\mathbf{A}}^{2}+\left(\mathbf{y}-\mathbf{x}_{j}, \mathbf{d}_{j}\right)_{\mathbf{A}}^{2} \\
\geq & \left\|\mathbf{x}_{j}-\mathbf{x}^{\star}\right\|_{\mathbf{A}}^{2}-\left(\mathbf{x}_{j}-\mathbf{x}^{\star}, \mathbf{d}_{j}\right)_{\mathbf{A}}^{2}
\end{aligned}
$$

$E_{j+1} \geq \delta_{\max } E_{j}$ now follows by taking expectation and applying Lemma 1 (notice that $\mathbf{y}$ is independent of $\mathbf{d}_{j}$ ),

Continuing along the lines of the proof of Theorem 2 (we omit the details), we have the following generalization of Theorem 2

Theorem 3. Consider iteration (8) with $\beta \leq 1$ for an arbitrary starting vector $\mathbf{x}_{0}$, that is iteration (4) where $\mathbf{d}_{0}, \mathbf{d}_{1}, \ldots$ are i.i.d. vectors that take $\mathbf{e}^{(1)}, \ldots, \mathbf{e}^{(n)}$ with equal probability, and $k(0), k(1), \ldots$ are such that $(6)$ holds but are independent of the random choices of $\mathbf{d}_{0}, \mathbf{d}_{1}, \ldots$ Let $\rho=\frac{1}{n}\|\mathbf{A}\|_{\infty}=\max _{l}\left\{\frac{1}{n} \sum_{r=1}^{n}\left|\mathbf{A}_{l r}\right|\right\}$. Provided that $2 \beta-$ $\beta^{2}-2 \rho \tau \beta^{2}>0$, the following holds:

(a) For every $m \geq \frac{\log (1 / 2)}{\log \left(1-\lambda_{\max } / n\right)} \approx \frac{0.693 n}{\lambda_{\max }}$ we have

$$
E_{m} \leq\left(1-\frac{\nu_{\tau}(\beta)}{2 \kappa}\right) E_{0}
$$

where

$$
\nu_{\tau}(\beta)=2 \beta-\beta^{2}-2 \rho \tau \beta^{2}
$$

(b) Let $T_{0}=\left\lceil\frac{\log (1 / 2)}{\log \left(1-\lambda_{\max } / n\right)}\right\rceil$ and $T=T_{0}+\tau$. For every $m \geq r T(r=1,2, \ldots)$ we have

$$
E_{m} \leq\left(1-\frac{\nu_{\tau}(\beta)}{2 \kappa}\right)\left(1-\frac{\nu_{\tau}(\beta)\left(1-\lambda_{\max } / n\right)^{\tau}}{2 \kappa}+\chi(\beta)\right)^{r-1} E_{0}
$$


where

$$
\chi(\beta)=\frac{\rho \tau^{2} \beta^{2} \lambda_{\max }\left(1-\lambda_{\max } / n\right)^{-2 \tau}}{n} .
$$

\section{Discussion:}

- We see that for a sufficiently small $\beta$ both bounds are useful, but the computation of the optimal $\beta$ for assertion (b) (in terms of the bound) requires some approximation of the condition number.

- Alternatively, we can optimize only the value of $\nu_{\tau}(\beta)$. The optimum of that term is achieved at $\tilde{\beta}=1 /(1+2 \rho \tau)$ and yields $\nu_{\tau}(\tilde{\beta})=1 /(1+2 \rho \tau)$. It is also the case that $\chi(\tilde{\beta})<\chi(1)$, so both bounds are improved. From a practical perspective, the challenge of setting the step size to $\tilde{\beta}$ is that $\tau$ might not be known. However, under normal circumstances (and in the reference scenario) we have $\tau=O(P)$, which can provide a general guideline for setting the step-size.

\section{Convergence Bound with Inconsistent Reads}

We now analyze the iteration under the inconsistent read model, i.e. iteration 9 .

Theorem 4. Consider iteration (9) for some $0 \leq \beta<1$ and an arbitrary starting vector $\mathbf{x}_{0}$, where $\mathbf{d}_{0}, \mathbf{d}_{1}, \ldots$ are i.i.d. vectors that take $\mathbf{e}^{(1)}, \ldots, \mathbf{e}^{(n)}$ with equal probability, and $K(0), K(1), \ldots$ are such that equation (7) holds but are independent of the random choices of $\mathbf{d}_{0}, \mathbf{d}_{1}, \ldots$ Let $\rho_{2}=\max _{l}\left\{\frac{1}{n} \sum_{r=1}^{n} \mathbf{A}_{l r}^{2}\right\}$. Provided that $\beta\left(1-\beta-\rho_{2} \tau^{2} \beta / 2\right)>$ 0 , the following holds:

(a) For every $m \geq \frac{\log (1 / 2)}{\log \left(1-\lambda_{\max } / n\right)} \approx \frac{0.693 n}{\lambda_{\max }}$ we have

$$
E_{m} \leq\left(1-\frac{\omega_{\tau}(\beta)}{2 \kappa}\right) E_{0}
$$

where

$$
\omega_{\tau}(\beta)=2 \beta\left(1-\beta-\rho_{2} \tau^{2} \beta / 2\right) .
$$

(b) Let $T_{0}=\left\lceil\frac{\log (1 / 2)}{\log \left(1-\lambda_{\max } / n\right)}\right\rceil$ and $T=T_{0}+\tau$. For every $m \geq r T(r=1,2, \ldots)$ we have

$$
E_{m} \leq\left(1-\frac{\omega_{\tau}(\beta)}{2 \kappa}\right)\left(1-\frac{\omega_{\tau}(\beta)\left(1-\lambda_{\max } / n\right)^{\tau}}{2 \kappa}+\psi(\beta)\right)^{r-1} E_{0}
$$

where

$$
\psi(\beta)=\frac{\rho_{2} \tau^{3} \beta^{2} \lambda_{\max }\left(1-\lambda_{\max } / n\right)^{-2 \tau}}{n} .
$$

Most of the proof is analogous to the proof of Theorem 2, so we give only a sketch that focuses on the unique parts.

Proof. (Sketch) As before:

$$
\left\|\mathbf{x}_{j+1}-\mathbf{x}^{\star}\right\|_{\mathbf{A}}^{2}=\left\|\mathbf{x}_{j}-\mathbf{x}^{\star}\right\|_{\mathbf{A}}^{2}-\beta(2-\beta)\left(\mathbf{x}_{K(j)}-\mathbf{x}^{\star}, \mathbf{d}_{j}\right)_{\mathbf{A}}^{2}-2 \beta\left(\mathbf{x}_{K(j)}-\mathbf{x}^{\star}, \mathbf{d}_{j}\right)_{\mathbf{A}}\left(\mathbf{x}_{j}-\mathbf{x}_{K(j)}, \mathbf{d}_{j}\right)_{\mathbf{A}} .
$$

We now bound the additional term: 


$$
\begin{aligned}
2 \beta\left(\mathbf{x}_{K(j)}-\mathbf{x}^{\star}, \mathbf{d}_{j}\right)_{\mathbf{A}}\left(\mathbf{x}_{j}-\mathbf{x}_{K(j)}, \mathbf{d}_{j}\right)_{\mathbf{A}} & =2 \beta\left(\mathbf{x}_{K(j)}-\mathbf{x}^{\star}, \mathbf{d}_{j}\right)_{\mathbf{A}}\left(\sum_{t \in K^{-}(j)} \beta \gamma_{t} \mathbf{d}_{t}, \mathbf{d}_{j}\right)_{\mathbf{A}} \\
& =2 \beta^{2}\left(\mathbf{x}_{K(j)}-\mathbf{x}^{\star}, \mathbf{d}_{j}\right)_{\mathbf{A}}\left(\sum_{t \in K^{-}(j)} \gamma_{t} \mathbf{d}_{t}, \mathbf{d}_{j}\right)_{\mathbf{A}} \\
\geq & -\beta^{2}\left[\left(\mathbf{x}_{K(j)}-\mathbf{x}^{\star}, \mathbf{d}_{j}\right)_{\mathbf{A}}^{2}+\left(\sum_{t \in K^{-}(j)} \gamma_{t} \mathbf{d}_{t}, \mathbf{d}_{j}\right)_{A}^{2}\right] \\
\geq & -\beta^{2}\left[\left(\mathbf{x}_{K(j)}-\mathbf{x}^{\star}, \mathbf{d}_{j}\right)_{\mathbf{A}}^{2}+\sum_{t \in K^{-}(j)}\left(\mathbf{x}_{K(t)}-\mathbf{x}^{\star}, \mathbf{d}_{t}\right)_{\mathbf{A}}^{2}\left(\mathbf{d}_{t}, \mathbf{d}_{j}\right)_{\mathbf{A}}^{2}\right] \\
\geq & -\beta^{2}\left[\left(\mathbf{x}_{K(j)}-\mathbf{x}^{\star}, \mathbf{d}_{j}\right)_{\mathbf{A}}^{2}+\tau \sum_{t \in K^{-}(j)}\left(\mathbf{x}_{K(t)}-\mathbf{x}^{\star}, \mathbf{d}_{t}\right)_{\mathbf{A}}^{2}\left(\mathbf{d}_{t}, \mathbf{d}_{j}\right)_{\mathbf{A}}^{2}\right]
\end{aligned}
$$

where $K^{-}(j)=\{0, \ldots, j-1\}-K(j)$. Since $\mathbf{x}_{K(t)}$ does not depend on $\mathbf{d}_{t}$ or $\mathbf{d}_{j}$, we can bound as before,

$$
\mathbb{E}\left[\left(\mathbf{x}_{K(t)}-\mathbf{x}^{\star}, \mathbf{d}_{t}\right)_{\mathbf{A}}^{2}\left(\mathbf{d}_{t}, \mathbf{d}_{j}\right)_{\mathbf{A}}^{2}\right] \leq \rho_{2} \mathbb{E}\left[\left(\mathbf{x}_{K(t)}-\mathbf{x}^{\star}, \mathbf{d}_{t}\right)_{\mathbf{A}}^{2}\right] .
$$

Therefore,

$$
E_{j+1} \leq E_{j}-2 \beta(1-\beta) \mathbb{E}\left[\left(\mathbf{x}_{K(j)}-\mathbf{x}^{\star}, \mathbf{d}_{j}\right)_{\mathbf{A}}^{2}\right]+\rho_{2} \tau \beta^{2} \sum_{t \in K^{-}(j)} \mathbb{E}\left[\left(\mathbf{x}_{K(t)}-\mathbf{x}^{\star}, \mathbf{d}_{t}\right)_{\mathbf{A}}^{2}\right] .
$$

After we unroll the recursion, we find that

$$
E_{k} \leq E_{0}-2 \beta\left(1-\beta-\rho_{2} \tau^{2} \beta / 2\right) \sum_{i=0}^{k-1} \mathbb{E}\left[\left(\mathbf{x}_{K(i)}-\mathbf{x}^{\star}, \mathbf{d}_{i}\right)_{\mathbf{A}}^{2}\right] .
$$

We can now continue to bound as in the proof of Theorem 2. The crucial observation is that $\mathbf{x}_{K(i)}$ is the result of $|K(i)|$ random single coordinate steps. So

$$
\mathbb{E}\left[\left\|\mathbf{x}_{K(i)}-\mathbf{x}^{\star}\right\|_{\mathbf{A}}^{2}\right] \geq \delta_{\max }^{|K(i)|} E_{0} \geq \delta_{\max }^{i} E_{0} .
$$

\section{Discussion:}

- Unlike the bounds for the consistent read model, the theorem guarantees convergence only for values of $\beta$ strictly smaller than 1.

- The bound has a worse dependence on $\tau$, so scalability is worse. The bound for consistent read has also a better dependence on the step-size $(\beta)$ in the sense that large step-sizes are allowed. In contrast, due to the unit diagonal assumption, we always have $\rho_{2} \leq \rho$, so the bound enjoys a more favorable dependence in that respect. However, we note that $\rho_{2} \geq 1 / n$, so if $\rho=O(1 / n)$ then the ratio between $\rho$ and $\rho_{2}$ is bounded by a constant, so the better dependence on $\tau$ makes the bound for consistent read more favorable (as we expect).

- The reason why we develop equation (19) instead of simply adapting equation (18) for the inconsistent read iteration is that the latter equation leads to expressions of the form $\left(\mathbf{x}_{K(j)}-\mathbf{x}^{\star}, \mathbf{d}_{j}\right)_{\mathbf{A}}^{2}\left|\left(\mathbf{d}_{t}, \mathbf{d}_{j}\right)_{\mathbf{A}}\right|$ for $t \in$ $\{0, \ldots, j-1\}-K(j)$. Such an expression is hard to analyze since $\mathbf{x}_{K(j)}$ can depend of $\mathbf{d}_{t}$. An example is $K(j)=\{0, \ldots, j-3, j-1\}$ and $t=j-2$ (for some $j \geq 3$ ). 


\section{Unsymmetric Systems and Overdetermined Least-Squares}

In this section, we consider the more general problem of finding the solution to $\min _{\mathbf{x}}\|\mathbf{A x}-\mathbf{b}\|_{2}$ where $\mathbf{A} \in \mathbb{R}^{r \times n}$ has at least as many rows as columns and is full rank. Note that this problem includes the solution of $\mathbf{A x}=\mathbf{b}$ for a general (possibly unsymmetric) non singular A. For simplicity, we will assume that the columns of $\mathbf{A}$ have unit Euclidean norm.

Lewis and Leventhal [11] analyzed this case as well, and suggest the following iteration

$$
\begin{aligned}
\mathbf{r}_{j} & =\mathbf{b}-\mathbf{A} \mathbf{x}_{j} \\
\gamma_{j} & =\mathbf{d}_{j}^{\mathrm{T}} \mathbf{A}^{\mathrm{T}} \mathbf{r}_{j} \\
\mathbf{x}_{j+1} & =\mathbf{x}_{j}+\gamma_{j} \mathbf{d}_{j}
\end{aligned}
$$

where $\mathbf{d}_{0}, \mathbf{d}_{1}, \ldots$ are i.i.d. random vectors, taking $\mathbf{e}^{(1)}, \ldots, \mathbf{e}^{(n)}$ with equal probability. One can show that this is a stochastic coordinate descent method on $f(\mathbf{x})=\|\mathbf{A} \mathbf{x}-\mathbf{b}\|_{2}$. Lewis and Leventhal prove that

$$
\mathbb{E}\left[\left\|\mathbf{A} \mathbf{x}_{m}-\mathbf{b}\right\|_{2}^{2}\right] \leq\left(1-\frac{\lambda_{\min }\left(\mathbf{A}^{\mathrm{T}} \mathbf{A}\right)}{n}\right)^{m}\left\|\mathbf{A} \mathbf{x}_{0}-\mathbf{b}\right\|_{2}^{2}
$$

where in the above $\lambda_{\min }\left(\mathbf{A}^{\mathrm{T}} \mathbf{A}\right)$ is the minimum eigenvalue of $\mathbf{A}^{\mathrm{T}} \mathbf{A}$.

It is rather straightforward to devise an asynchronous version of the algorithm, following the same strategy we used for AsyRGS. We only note that traditional presentations of (20) favor keeping a residual vector $\mathbf{r}$ in memory, and updating after each update on $\mathbf{x}$. However, updates to $\mathbf{r}$ cannot be atomic, so in an asynchronous version of the iteration, the necessary entries of the residual (i.e., the non-zero indices of $\mathbf{A} \mathbf{d}_{j}$ ) have to be computed in each iteration. Introducing a step-size $\beta$ as well, leads to the following iteration that describes the asynchronous algorithm (inconsistent read):

$$
\begin{aligned}
\gamma_{j} & =\mathbf{d}_{j}^{\mathrm{T}} \mathbf{A}^{\mathrm{T}}\left(\mathbf{b}-\mathbf{A} \mathbf{x}_{K(j)}\right) \\
\mathbf{x}_{j+1} & =\mathbf{x}_{j}+\beta \gamma_{j} \mathbf{d}_{j}
\end{aligned}
$$

We remark that each iteration of the asynchronous algorithm that implements (21) is more expensive then the best implementation of 20]. The main cost per step of the asynchronous algorithm is in computing $\gamma_{j}$. Suppose that the row vector $\mathbf{d}_{j}^{\mathrm{T}} \mathbf{A}^{\mathrm{T}}$ (which is simply a transposed column of $\mathbf{A}$ ) has $l_{j}$ non-zeros, and that the rows corresponding the non-zero indices in $\mathbf{d}_{j}^{\mathrm{T}} \mathbf{A}^{\mathrm{T}}$ have $r_{j, 1}, \ldots, r_{j, l_{j}}$ non-zeros. The cost of iteration $j$ of [21] is then $O\left(\sum_{i=1}^{l_{j}} r_{j, i}\right)$. As explained, for (20), we can have cheaper steps by keeping both $\mathbf{x}$ and $\mathbf{r}$ in memory. Each iteration then updates both $\mathbf{x}$ and $\mathbf{r}$ leading to a cost per iteration of $O\left(l_{j}\right)$. Since all rows have at least one non-zero, this is obviously superior. It is important to note that while for some matrices the additional cost might be disastrous, it is not necessarily the case. For example, if the number of non-zeros in each column and row is bounded between $C_{1}$ and $C_{2}$, each iteration is at most $O\left(C_{2}^{2} / C_{1}\right)$ more expensive. If both $C_{2}$ and $C_{2} / C_{1}$ are small, this ratio might be small enough to be overcome by a sufficient amount of processors (in particular, if $C_{1}=O(1)$ and $C_{2}=O(1)$ then the asymptotic cost per iteration is unaffected).

We also remark that for 21 to be a valid description of the behavior of the algorithm, care has to be taken that in each iteration each entry of $\mathbf{x}$ that is read, is read only once.

Notice that 21) is identical to the iteration of AsyRGS on $\mathbf{A}^{\mathrm{T}} \mathbf{A x}=\mathbf{A}^{\mathrm{T}} \mathbf{b}$. This leads immediately to the following theorem.

Theorem 5. Consider iteration (21) for some $0 \leq \beta<1$ and an arbitrary starting vector $\mathbf{x}_{0}$, where $\mathbf{d}_{0}, \mathbf{d}_{1}, \ldots$ are i.i.d. vectors that take $\mathbf{e}^{(1)}, \ldots, \mathbf{e}^{(n)}$ with equal probability, and $K(0), K(1), \ldots$ are such that equation (7) holds but are independent of the random choices of $\mathbf{d}_{0}, \mathbf{d}_{1}, \ldots$ Let $\mathbf{X}=\mathbf{A}^{\mathrm{T}} \mathbf{A}$ and let $\rho_{2}=\max _{l}\left\{\frac{1}{n} \sum_{r=1}^{n} \mathbf{X}_{l r}^{2}\right\}$. Let $\mathbf{x}^{\star}=\arg \min _{\mathbf{x}}\|\mathbf{A} \mathbf{x}-\mathbf{b}\|_{2}$. Denote by $\kappa$ the condition number of $\mathbf{A}$ (ratio between largest and smallest singular values of $\mathbf{A})$ and by $\sigma_{\max }$ the maximum singular value of $\mathbf{A}$. Provided that $\beta\left(1-\beta-\rho_{2} \tau^{2} \beta / 2\right)>0$, the following holds: 
(a) For every $m \geq \frac{\log (1 / 2)}{\log \left(1-\sigma_{\max }^{2} / n\right)} \approx \frac{0.693 n}{\sigma_{\max }^{2}}$ we have

$$
\mathbb{E}\left[\left\|\mathbf{x}_{m}-\mathbf{x}^{\star}\right\|_{\mathbf{X}}^{2}\right] \leq\left(1-\frac{\omega_{\tau}(\beta)}{2 \kappa^{2}}\right)\left\|\mathbf{x}_{0}-\mathbf{x}^{\star}\right\|_{\mathbf{X}}^{2}
$$

where

$$
\omega_{\tau}(\beta)=2 \beta\left(1-\beta-\rho_{2} \tau^{2} \beta / 2\right)
$$

(b) Let $T_{0}=\left\lceil\frac{\log (1 / 2)}{\log \left(1-\sigma_{\max }^{2} / n\right)}\right\rceil$ and $T=T_{0}+\tau$. For every $m \geq r T(r=1,2, \ldots)$ we have

$$
\mathbb{E}\left[\left\|\mathbf{x}_{m}-\mathbf{x}^{\star}\right\|_{\mathbf{X}}^{2}\right] \leq\left(1-\frac{\omega_{\tau}(\beta)}{2 \kappa^{2}}\right)\left(1-\frac{\omega_{\tau}(\beta)\left(1-\sigma_{\max }^{2} / n\right)^{\tau}}{2 \kappa^{2}}+\chi(\beta)\right)^{r-1}\left\|\mathbf{x}_{0}-\mathbf{x}^{\star}\right\|_{\mathbf{X}}^{2}
$$

where

$$
\chi(\beta)=\frac{\rho_{2} \tau^{3} \beta^{2} \sigma_{\max }^{2}\left(1-\sigma_{\max }^{2} / n\right)^{-2 \tau}}{n} .
$$

Note that when $\mathbf{A} \mathbf{x}^{\star}=\mathbf{b}$ we have $\left\|\mathbf{x}_{m}-\mathbf{x}^{\star}\right\|_{\mathbf{X}}^{2}=\left\|\mathbf{A} \mathbf{x}_{m}-\mathbf{b}\right\|_{2}^{2}$ and $\left\|\mathbf{x}_{0}-\mathbf{x}^{\star}\right\|_{\mathbf{X}}^{2}=\left\|\mathbf{A} \mathbf{x}_{0}-\mathbf{b}\right\|_{2}^{2}$.

\section{Experiments}

The main goals of this section are threefold. First, we show that the proposed algorithm can be advantageous for certain types of linear systems, such as those arising in the analysis of big data, even in the absence of massive parallelism. Secondly, we explore the behavior of the algorithm in terms of scalability and the penalty paid for asynchronicity for these type of matrices. Finally, we demonstrate that our algorithm can serve as an effective preconditioner for a flexible Krylov method.

It is not the goal of this section to show that the suggested algorithm converges faster than standard algorithms like CG for all, or many, matrices. The synchronous method on which our algorithm is based requires $O(\kappa \cdot n)$ iterations for convergence, which are equivalent to about $O(\kappa)$ CG iterations. In comparison, CG converges at a much better rate of $O(\sqrt{\kappa})$. Therefore, for a general purpose solver, our algorithm might be advantageous only as a preconditioner in a Krylov subspace method that can handle a preconditioner that changes from one step to another. Such methods are known as flexible Krylov subspace methods [17, 16, 19]. While we explore this combination, it is not the purpose of this paper to present a general purpose production-grade linear solver. For such a solver, sophisticated rules for setting the preconditioner parameters, and various heuristics will be needed to avoid a completely random access pattern which is likely to cause poor performance due to extensive cache misses, just to name a few of the issues that need to be resolved for a production-grade linear solver. Exploration of these issues is slated for continued future research, and is outside the scope of this paper.

We experiment with a linear system arising from performing linear regression to analyze social media data. The system arises from a real-life data analysis task performed on real data. The matrix A is 120,147 × 120,147 (after removing rows and columns that were identically zero) and it has $172,892,749$ non-zeros. The matrix is generated by computing the Gram matrix of a data matrix in which each row corresponds to a text document and the values are the term frequencies inside the document. The maximum number of non-zeros in a row is 117,182, so some rows are almost full. However, the row sizes are highly skewed as the average number of non-zeros in a row is 1439 , and the minimum is 1 . The right-hand side $\mathbf{B}$ has 51 columns, each corresponding to a label prediction. For both CG and our algorithm, the 120,147 $\times 51$ right-hand side and solution matrices are stored in a row-major fashion to improve locality. All 51 systems are solved together. We initialize the solution to an all zero matrix. The matrix does not have unit-diagonal, so for Randomized Gauss-Seidel and AysRGS we use iteration (3). The coefficient matrix has very little to no structure. This implies that reordering $\mathbf{A}$ in order to reduce cache misses in the matrix-vector multiplications has very little effect. Luckily, the downstream application requires very low accuracy. In particular, running Randomized 


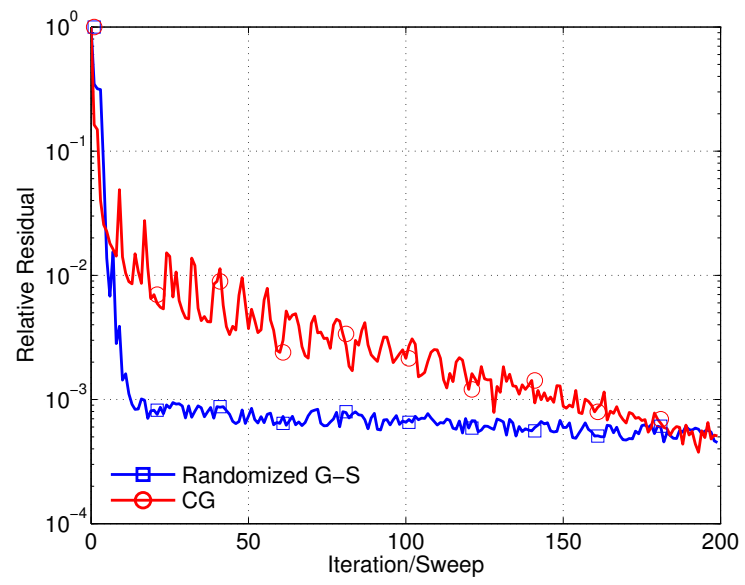

Figure 1: Residual of Randomized Gauss-Seidel and CG on the test matrix.

Gauss-Seidel beyond 10 sweeps has negligible improvement in the downstream use (as measured in the application specific metric), even though the residual continues to drop.

For this matrix $\rho \approx 231 / n$ and $\rho_{2} \approx 8.9 / n$, so the parameters $\nu_{\tau}$ and $\omega_{\tau}$ that govern the slowdown with occasional synchronization are not too bad (e.g., $\nu_{200}(1.0)=0.618$ and $\left.\omega_{200}(0.25)=0.1906\right)$. However matrix is highly ill-conditioned (as verified using an iterative condition-number estimator [2]) so the bounds without occasional synchronization do not apply unless we set the step size extremely small. In that respect, we note that the theoretical bounds for the synchronous algorithm are already far from being descriptive of the behavior of that algorithm, at least for a small amount of sweeps.

We remark that we chose to experiment with this matrix even though it does not fit the reference scenario, which was the main focus of our theoretical analysis. We wanted to experiment with an application for which our algorithm makes sense as a standalone algorithm, instead of the case where it is applied as a building block in a larger solver, which is the case for most matrices from scientific computing applications. In addition, we note that the high imbalance in the row size only makes this matrix more challenging for an asynchronous solver (the maximum delay $\tau$ might be large). The analysis presented in this paper is not able to cover effectively such high variance in row sizes, but we conjecture that the results can be strengthened to cover it, and propose a strategy in the conclusions. Finally, by choosing this test case, we are able to show that in practice, the algorithm can be suitable even for situations outside the scope of our analysis.

Figure 1 1 plots the residual ( $\left.\|\mathbf{A X}-\mathbf{B}\|_{F} /\|\mathbf{B}\|_{F}\right)$ of Randomized Gauss-Seidel and CG as the iterations progress. We see that Randomized Gauss-Seidel initially progresses faster than CG. This suggests that Randomized GaussSeidel, and its asynchronous variants, might be well suited as a preconditioner in a flexible Krylov method. We remark that the behavior of $\mathrm{CG}$ can be improved with preconditioning.

We tested parallel performance on a single BlueGene/Q node. The compute node has 16 compute cores (and an additional one for services) running at $1.6 \mathrm{GHz}$, each capable of 4-way multithreading. We experimented with the inconsistent read variant only. In Figure 2 (left), we plot the running time of 10 iterations (sweeps) of AsyRGS and CG. We use a SIMD variant of CG where the indices are assigned to threads in a round-robin manner. The use of round-robin is due to the fact that the coefficient matrix has very little to no structure, so other distribution methods give very little benefit while incurring a large overhead. We see that AsyRGS shows almost linear scalability, and attains a speedup of almost 48 on 64 threads. CG initially shows good speedups as well, but strays from linear speedup as the thread count grows. In the serial run, Randomized Gauss-Seidel was about $10 \%$ faster (1220 seconds versus 1330 seconds for CG). With 64 threads the gap is substantial: 25.7 seconds versus 46.5 seconds for CG. The speedup of CG on 64 threads is less than 29.

Next, we explore whether there is a price, in terms of the final residual, for using an asynchronous version. To that 

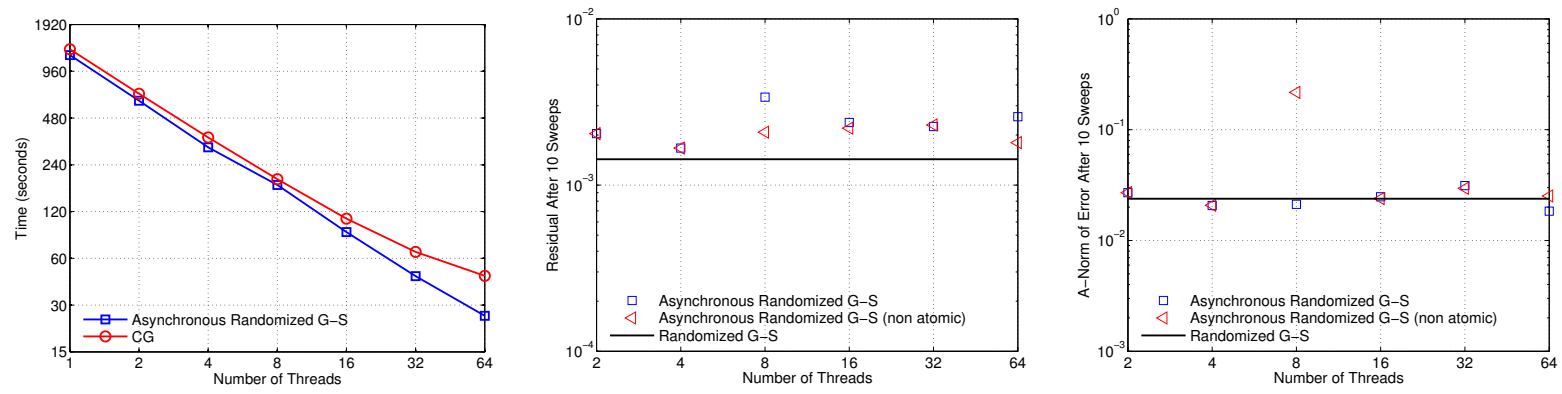

Figure 2: Performance of AsyRGS (inconsistent read) on the test matrix. Left: Running time of both AsyRGS and CG on the test matrix. Center: Relative residual after 10 sweeps of Randomized Gauss-Seidel and AsyRGS. Right: Relative A-norm of the error.

Table 1: Performance of Flexible-CG with AsyRGS (inconsistent read) serving as a preconditoner. We explore the performance when varying the number of inner (preconditioner) sweeps. 64 threads are used. As runs are not deterministic, we report the median of five different runs.

\begin{tabular}{|c|c|c|c|c|}
\hline Inner sweeps & Outer iterations & Outer $\times($ Inner + 1) & Time & Mat-ops / sec \\
\hline \hline 30 & 38 & 1178 & $234 \mathrm{sec}$ & 5.03 \\
\hline 20 & 48 & 1008 & $203 \mathrm{sec}$ & 4.97 \\
\hline 10 & 69 & 759 & $159 \mathrm{sec}$ & 4.77 \\
\hline 5 & 100 & 600 & $132 \mathrm{sec}$ & 4.55 \\
\hline 3 & 151 & 604 & $134 \mathrm{sec}$ & 4.51 \\
\hline $\mathbf{2}$ & $\mathbf{1 8 4}$ & $\mathbf{5 5 2}$ & $\mathbf{1 2 5} \mathbf{~ s e c}$ & $\mathbf{4 . 4 2}$ \\
\hline 1 & 356 & 712 & $162 \mathrm{sec}$ & 4.39 \\
\hline
\end{tabular}

end, we made sure that the set of directions $\mathbf{d}_{0}, \mathbf{d}_{1}, \ldots$ is fixed using the library Random123 [18] which allows random access to the pseudo-random numbers, as opposed to the conventional streamed approach. Here we also try a variant of AsyRGS which does not perform atomic writes, in order to test experimentally whether atomic writes are necessary (from a theoretical point-of-view, so far we have not been able to analyze the convergence rate without atomic writes). In Figure22(center), we plot the residual after 10 sweeps of a single run on each thread count. We see that the residual of the asynchronous algorithm is slightly worse than that of the synchronous method, although it is of the same order of magnitude. There does not seem to be a consistent advantage to using atomic writes. There is also variation in the residual due to different scheduling of the threads, so we conducted 5 additional trials with 64 threads. The minimum residual of AsyRGS was $1.44 \times 10^{-3}$ and the maximum was $2.88 \times 10^{-3}$. With the non-atomic variant, the minimum was $1.39 \times 10^{-3}$ and the maximum was $2.96 \times 10^{-3}$. There is no noticeable difference between the running time of the two variants.

In Figure 2 (right) we examine the relative $\mathbf{A}$-norm of the error $\left(\left\|\mathbf{x}-\mathbf{x}^{\star}\right\|_{\mathbf{A}} /\left\|\mathbf{x}^{\star}\right\|_{\mathbf{A}}\right)$ after 10 sweeps for different thread counts. In this experiment, we use a single right hand side that is generated as follows: we took one of the right hand sides of the original problem, solved to very low residual (using Flexible-CG, as explained in the following paragraphs) to form $\mathbf{x}^{\star}$, and then used $\mathbf{b}=\mathbf{A} \mathbf{x}^{\star}$. We see that the A-norm of the error for AsyRGS is very close to the A-norm of the error of the synchronous method, and is sometimes better (we caution that we did a single experiment, and there is variation in the error produced by the asynchronous method). Both the synchronous and the asynchronous method produce errors that are well below the theoretical bounds for the synchronous method.

Our final set of experiments explore the use of AsyRGS as a preconditioner for a flexible Krylov method. We implemented Notay's Flexible-CG algorithm [16]. In our implementation we do not use truncation or restarts, although we acknowledge that a general purpose production-grade solver might require these. We compute the norm of the 

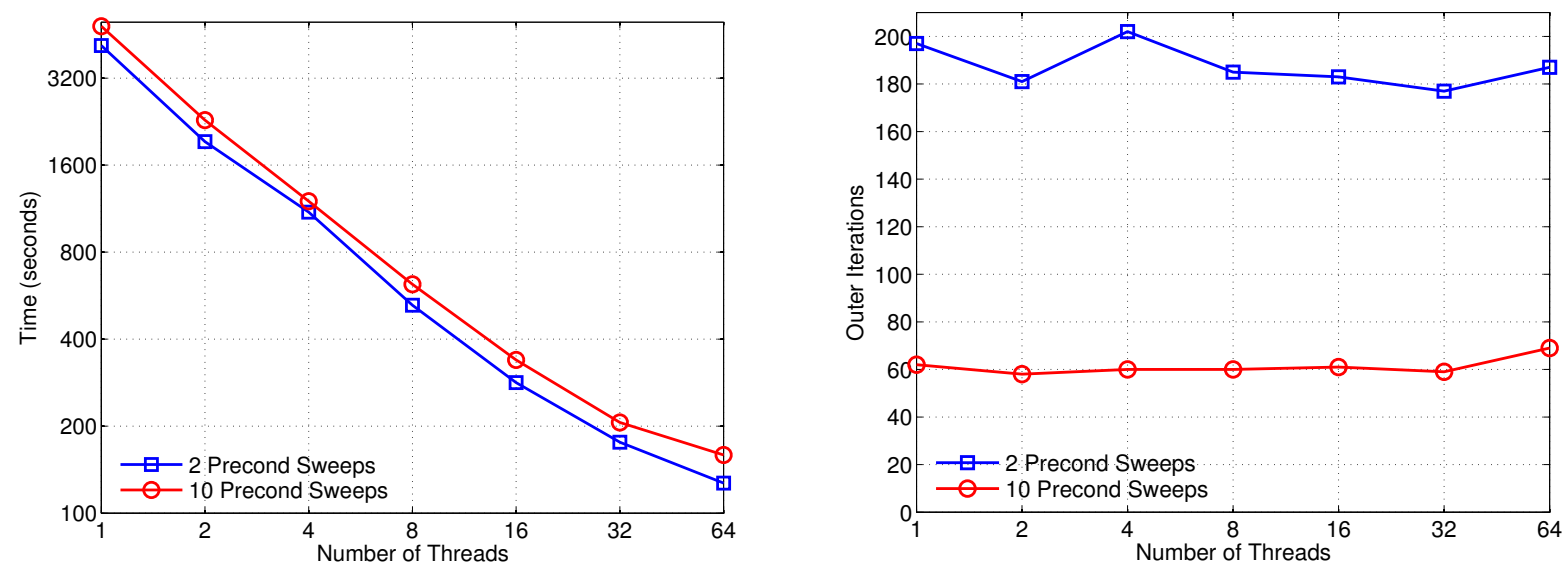

Figure 3: Parallel performance of Flexible-CG preconditioned using AsyRGS (inconsistent read) on the test matrix. Left: Running time of the algorithm, with 2 or 10 sweeps per preconditioner application, on the test matrix. Right: Number of outer (Flexible-CG) iterations.

residual after every iteration of Flexible-CG, and declare convergence once the relative residual has dropped below some predefined threshold. In our experiments we use $10^{-8}$. Iteration counts and running time are reported based on convergence. We use AsyRGS as the preconditioner, with the number of sweeps set as a parameter. As AsyRGS introduces non-determinism, we repeat every experiment five times, and report the median (we note that the random choices are fixed in these five runs, and non-determinism is only due to asynchronism). We use only a single right-hand side vector in this set of experiments.

There is a trade-off in setting the number of AsyRGS sweeps to use as a preconditiner. As we increase the number of preconditioner sweeps, the preconditioner improves so we expect the number of outer (Flexible-CG) iterations to decrease. On the other hand, AsyRGS is not as effective on utilizing residual information as CG, which enjoys a superior converge rate compared to Randomized Gauss-Seidel, so we expect the number of times the matrix is operated on, which is equal to OuterIterations $\times($ InnerSweeps +1$)$, to increase as we increase the number of preconditioner sweeps. On the flip side, as we increase the number of preconditioner sweeps, we expect parallel efficiency to increase as well since we are diverting work to AsyRGS, which enjoys better parallel efficiency. Table 1 explores this tradeoff. In this experiment we vary the number of inner preconditioner sweeps, and run Flexible-CG to convergence. 64 threads are used. As we expect, the number of outer iterations decreases as the number of inner sweeps increases, but the overall times the matrix is operated on increases (with the notable exception of using a single inner sweep). Improved efficiency is demonstrated in the "Mat-ops / sec" columns (which is equal to OuterIterations $\times$ (InnerSweeps $+1)$ / Time): we see that as the number of inner sweeps increase, we operate on the matrix at a higher rate. Nevertheless, the optimal number of sweeps (in terms of running time) is obtained with only two inner sweeps.

Parallel performance is explored in Figure 3. In the left graph we plot the running time as a function of the number of threads, for two configurations: 2 inner sweeps and 10 inner sweeps. We see that the method exhibits good speedups, with speedup of more than 32 for 2 inner sweeps, and 30 for 10 inner sweeps. On the surface it appears that the two inner sweeps configuration enjoys better scalability, which is counter to our intuition that diverting more work to the asynchronous iterations should improve parallel efficiency. However, the running time is measured until convergence, and the number of (outer) iterations is also a function of the number of threads. This is explored in the right graph of Figure 3. While intuitively the number of iterations should grow with the number of threads, as the quality of the preconditioner should degrade due to increased asynchronism, that is not observed in practice. We do see higher variability in the number of iterations with 2 inner sweeps. Possibly the reason for these observations is that the random choices made by the algorithm are more dominant than asynchronism in determining convergence. We now note that the speedup in terms of mat-ops / sec for 10 inner sweeps is almost 34, versus only 28 for 2 inner sweeps, which is 
consistent with the intuition that diverting more work to the asynchronous iterations should improve parallel efficiency.

\section{Conclusions and Future Work}

As we push forward toward exascale systems, it is becoming imperative to revisit asynchronous linear solvers as a means of addressing the limitations foreseen by current hardware trends. This paper serves as a starting point for this revisit. Our main observation is that the limitations of previous asynchronous linear solvers can be addressed by a new class of asynchronous methods based on randomization. Our analytical results clearly show the advantage of using randomization as a building block for asynchronous solvers.

While we do present experimental results that show the usefulness of our algorithm for certain types of linear systems, it is also clear that much needs to be done for a general purpose solver. One clear path, which we only started to explore, is the use of our algorithm as a preconditioner in a flexible Krylov method. Another, is to extend our algorithm from a shared memory system with limited parallelism to massively parallel systems.

There are some theoretical questions that need to be explored too. Is that gap in the bound for consistent and inconsistent reads inherent, or an improved analysis can remove or narrow it? Is it possible to obtain comparable bounds when we allow $k(j)$ or $K(j)$ to depend on $\mathbf{d}_{0}, \ldots, \mathbf{d}_{j}$ ? In our reference scenario, we show weak-scaling only if we periodically synchronize the threads. It is worth investigating whether the periodic synchronization is essential, or if it is an artifact of the analysis. In addition, in our analysis the convergence rate depends on the maximum age of data used during the algorithm. The maximum can be rather large in some setups (e.g., high ratio between maximum and minimum amount of non-zeros per row), but the use of the maximum is also rather pessimistic (the analysis assumes that the maximum delay is almost achieved). Perhaps a probabilistic modeling of the delays might lead to a convergence result that will be more descriptive for matrices with imbalanced row sizes.

\section{Acknowledgments}

Thanks to Vikas Sindhwani for providing the matrix used in the experiments. Haim Avron acknowledges the support from the XDATA program of the Defense Advanced Research Projects Agency (DARPA), administered through Air Force Research Laboratory contract FA8750-12-C-0323.

\section{References}

[1] H. Avron, A. Druinsky, and A. Gupta. Revisiting asynchronous linear solvers: Provable convergence rate through randomization. In Proceeding of the 28th IEEE International Parallel \& Distributed Processing Symposium (IPDPS), 2014.

[2] Haim Avron, Alex Druinsky, and Sivan Toledo. Reliable iterative condition-number estimation. CoRR, abs/1301.1107, 2013.

[3] G. M. Baudet. Asynchronous iterative methods for multiprocessors. J. ACM, 25(2):226-244, April 1978.

[4] D. P. Bertsekas and J. N. Tsitsiklis. Parallel and distributed computation. Prentice Hall, 1989.

[5] I. Bethune, J. M. Bull, N. J. Dingle, and N. J. Higham. Performance analysis of asynchronous Jacobi's method implemented in MPI, SHMEM and OpenMP. MIMS EPrint 2012.62, University of Manchester, UK, June 2012.

[6] D. Chazan and W. Miranker. Chaotic relaxation. Linear Algebra and its Applications, 2(2):199 - $222,1969$.

[7] N .M. Freris and A. Zouzias. Fast distributed smoothing for network clock synchronization. In IEEE Conference on Decision and Control, 2012. 
[8] A. Frommer and D. B. Szyld. On asynchronous iterations. Journal of Computational and Applied Mathematics, 123:201 - 216, 2000.

[9] M. Griebel and P. Oswald. Greedy and randomized versions of the multiplicative Schwarz method. Linear Algebra and its Applications, 437(7):1596 - 1610, 2012.

[10] J. Hook and N. J. Dingle. Performance analysis of asynchronous parallel Jacobi. MIMS EPrint 2013.52, University of Manchester, UK, October 2013.

[11] D. Leventhal and A. S. Lewis. Randomized methods for linear constraints: Convergence rates and conditioning. Math. Oper. Res., 35(3):641-654, 2010.

[12] J. Liu and S. J. Wright. Asynchronous Stochastic Coordinate Descent: Parallelism and Convergence Properties. SIAM Journal of Optimization, to Appear, 2014.

[13] J. Liu, S. J. Wright, C. Re, V. Bittorf, and S. Sridhar. An asynchronous parallel stochastic coordinate descent algorithm. In International Conference in Machine Learning (ICML), 2014.

[14] J. Liu, S. J. Wright, and S. Sridhar. An Asynchronous Parallel Randomized Kaczmarz Algorithm. ArXiv e-prints, January 2014.

[15] F. Niu, B. Recht, C. Re, and S. J. Wright. Hogwild: A lock-free approach to parallelizing stochastic gradient descent. In Advances in Neural Information Processing Systems (NIPS) 24, pages 693-701, 2011.

[16] Y. Notay. Flexible conjugate gradients. SIAM Journal on Scientific Computing, 22(4):1444-1460, 2000.

[17] Y. Saad. A flexible inner-outer preconditioned GMRES algorithm. SIAM Journal on Scientific Computing, 14(2):461-469, 1993.

[18] J. K. Salmon, M. A. Moraes, R. O. Dror, and D. E. Shaw. Parallel random numbers: as easy as 1, 2, 3. In Proceedings of SC'11, pages 16:1-16:12. ACM, 2011.

[19] V. Simoncini and D. B. Szyld. Flexible inner-outer Krylov subspace methods. SIAM Journal on Numerical Analysis, 40(6):2219-2239, 2003.

[20] T. Strohmer and R. Vershynin. A randomized Kaczmarz algorithm with exponential convergence. Journal of Fourier Analysis and Applications, 15:262-278, 2009. 